TRANSACTIONS OF THE

AMERICAN MATHEMATICAL SOCIETY

Volume 355 , Number 5 , Pages 2065-2095

S 0002-9947(03)03211-2

Article electronically published on January 10, 2003

\title{
HEAT KERNELS ON METRIC MEASURE SPACES AND AN APPLICATION TO SEMILINEAR ELLIPTIC EQUATIONS
}

\author{
ALEXANDER GRIGOR'YAN, JIAXIN HU, AND KA-SING LAU
}

\begin{abstract}
We consider a metric measure space $(M, d, \mu)$ and a heat kernel $p_{t}(x, y)$ on $M$ satisfying certain upper and lower estimates, which depend on two parameters $\alpha$ and $\beta$. We show that under additional mild assumptions, these parameters are determined by the intrinsic properties of the space $(M, d, \mu)$. Namely, $\alpha$ is the Hausdorff dimension of this space, whereas $\beta$, called the walk dimension, is determined via the properties of the family of Besov spaces $W^{\sigma, 2}$ on $M$. Moreover, the parameters $\alpha$ and $\beta$ are related by the inequalities $2 \leq \beta \leq \alpha+1$.

We prove also the embedding theorems for the space $W^{\beta / 2,2}$, and use them to obtain the existence results for weak solutions to semilinear elliptic equations on $M$ of the form

$$
-\mathcal{L} u+f(x, u)=g(x),
$$
\end{abstract}

where $\mathcal{L}$ is the generator of the semigroup associated with $p_{t}$.

The framework in this paper is applicable for a large class of fractal domains, including the generalized Sierpiński carpet in $\mathbb{R}^{n}$.

\section{INTRODUCTION}

We say that a triple $(M, d, \mu)$ is a metric measure space if $(M, d)$ is a nonempty metric space and $\mu$ is a Borel measure on $M$. Given a metric measure space $(M, d, \mu)$, a family $\left\{p_{t}\right\}_{t>0}$ of non-negative measurable functions $p_{t}(x, y)$ on $M \times M$ is called a heat kernel or a transition density if the following conditions are satisfied, for all $x, y \in M$ and $s, t>0$ :

(1) Symmetry: $p_{t}(x, y)=p_{t}(y, x)$.

(2) Normalization (or stochastic completeness):

$$
\int_{M} p_{t}(x, y) d \mu(y)=1
$$

(3) Semigroup property:

$$
p_{s+t}(x, y)=\int_{M} p_{s}(x, z) p_{t}(z, y) d \mu(z) .
$$

Received by the editors July 23, 2002.

2000 Mathematics Subject Classification. Primary 60J35; Secondary 28A80, 35J60.

The first author was partially supported by a visiting grant of the Institute of Mathematical Sciences of CUHK (the Chinese University of Hong Kong). The second author was supported by a Postdoctoral Fellowship from CUHK. The third author was partially supported by a HKRGC grant at CUHK.

(C) 2003 by A. Grigor'yan, J. Hu, and K.-S. Lau 
(4) Identity approximation: for any $f \in L^{2}(M, \mu)$,

$$
\int_{M} p_{t}(x, y) f(y) d \mu(y) \stackrel{L^{2}(M, \mu)}{\longrightarrow} f(x) \quad \text { as } t \rightarrow 0+.
$$

For example, the classical Gauss-Weierstrass function in $\mathbb{R}^{n}$,

$$
p_{t}(x, y)=\frac{1}{(4 \pi t)^{n / 2}} \exp \left(-\frac{|x-y|^{2}}{4 t}\right),
$$

satisfies this definition. Another elementary example is the Cauchy-Poisson kernel in $\mathbb{R}^{n}$ :

$$
p_{t}(x, y)=\frac{C_{n}}{t^{n}}\left(1+\frac{|x-y|^{2}}{t^{2}}\right)^{-\frac{n+1}{2}}
$$

where $C_{n}=\Gamma\left(\frac{n+1}{2}\right) / \pi^{(n+1) / 2}$.

Any heat kernel gives rise to the heat semigroup $\left\{T_{t}\right\}_{t>0}$ where $T_{t}$ is an operator in $L^{2}(M, \mu)$ defined by

$$
T_{t} u(x)=\int_{M} p_{t}(x, y) u(y) d \mu(y) .
$$

The above properties of $p_{t}$ imply that $T_{t}$ is a bounded selfadjoint operator in $L^{2}(M, \mu)$; moreover, $\left\{T_{t}\right\}_{t>0}$ is a strongly continuous, positivity-preserving, contraction semigroup in $L^{2}(M, \mu)$. Another way of constructing such a semigroup is to set

$$
T_{t}=\exp (t \mathcal{L})
$$

where $\mathcal{L}$ is a non-positive-definite selfadjoint operator in $L^{2}(M, \mu)$ satisfying in addition the Markov property. Typically, $-\mathcal{L}$ arises as the generator of a Dirichlet form. It is not always the case that the semigroup $\left\{T_{t}\right\}$ defined by (1.4) possesses an integral kernel. If it does, then the integral kernel will be a heat kernel in the above sense (although some additional restrictions are needed to ensure the normalization condition).

In this note we would like to adopt the axiomatic approach to heat kernels, which to some extent is opposite to the above scheme. Namely, we will assume that a heat kernel is defined on a metric measure space, and show that this implies many interesting consequences for analysis on such a space. A similar approach was used by M. Barlow [3] and K. Pietruska-Pałuba [23], although in their works a heat kernel was assumed to be the transition density of a diffusion process on $M$, and in [23] the underlying space $M$ was a subset of $\mathbb{R}^{n}$.

Let a heat kernel $p_{t}$ on $(M, d, \mu)$ satisfy the following two-sided estimate, for $\mu$-almost all $x, y \in M$ and all $t \in(0, \infty)$ :

$$
\frac{1}{t^{\alpha / \beta}} \Phi_{1}\left(\frac{d(x, y)}{t^{1 / \beta}}\right) \leq p_{t}(x, y) \leq \frac{1}{t^{\alpha / \beta}} \Phi_{2}\left(\frac{d(x, y)}{t^{1 / \beta}}\right),
$$

where $\alpha, \beta$ are positive constants, and $\Phi_{1}$ and $\Phi_{2}$ are non-negative monotone decreasing functions on $[0,+\infty)$.

For example, the Gauss-Weierstrass heat kernel (1.1) satisfies (1.5) with $\alpha=n$, $\beta=2$, and

$$
\Phi_{1}(s)=\Phi_{2}(s)=\frac{1}{(4 \pi)^{n / 2}} \exp \left(-\frac{s^{2}}{4}\right)
$$


The Cauchy-Poisson heat kernel (1.2) satisfies (1.5) with $\alpha=n, \beta=1$, and

$$
\Phi_{1}(s)=\Phi_{2}(s)=C_{n}\left(1+s^{2}\right)^{-\frac{n+1}{2}} .
$$

Note that the Gauss-Weierstrass heat kernel is generated by the classical Laplace operator in $\mathbb{R}^{n}$,

$$
-\Delta=-\sum_{k=1}^{n} \frac{\partial^{2}}{\partial x_{k}^{2}},
$$

whereas the Cauchy-Poisson heat kernel is generated by the operator $-(-\Delta)^{1 / 2}$. More generally, for any $0<\beta<2$ the operator $-(-\Delta)^{\beta / 2}$ generates a symmetric stable process of index $\beta$ whose heat kernel satisfies the estimate (1.5) with $\alpha=n$ and the following functions $\Phi_{1}$ and $\Phi_{2}$ :

$$
\Phi_{i}(s)=c_{i}\left(1+s^{2}\right)^{-\frac{\alpha+\beta}{2}},
$$

where $c_{i}$ is a positive constant, $i=1,2$.

The development of analysis on fractals has brought plenty of examples of heat kernels satisfying (1.5) with functions $\Phi_{1}$ and $\Phi_{2}$ of the form

$$
\Phi_{i}(s)=c_{i}^{\prime} \exp \left(-c_{i}^{\prime \prime} s^{\gamma}\right),
$$

where $\gamma>0$, and $c_{i}^{\prime}, c_{i}^{\prime \prime}$ are positive constants. In these examples the parameter $\beta$ is typically larger than 2 .

The nature of the parameters $\alpha$ and $\beta$ is of great interest. Although originally they are defined through the heat kernel, a posteriori they happen to be the invariants of the space $(M, d, \mu)$ itself, provided the function $\Phi_{2}$ decays at $\infty$ sufficiently fast. The parameter $\alpha$ happens to be the Hausdorff dimension of $M$ (see also [10]). The nature of the parameter $\beta$ is more complicated. We will call it the walk dimension of the heat kernel $p_{t}$. This terminology is motivated by the following observation: if the heat kernel $p_{t}$ is the transition density of a Markov process $X_{t}$ on $M$, then (1.5) implies (under reasonable assumptions about $\Phi_{1}$ and $\Phi_{2}$ ) that the mean time $t$ needed for the process $X_{t}$ to move away to a distance $r$ from the origin is of the order $r^{\beta}$ (see, for example, [3] Lemma 3.9]).

In this paper, we adopt an analytic approach, which does not depend on the existence of the process $X_{t}$. Following [18, we introduce on $(M, d, \mu)$ the family $\left\{W^{\sigma, 2}\right\}_{\sigma>0}$ of Besov spaces, which generalizes the Sobolev space $W^{1,2}$ in $\mathbb{R}^{n}$ (see Section 4; note that in the notation of [18], $\left.W^{\sigma, 2}=\operatorname{Lip}(\sigma, 2, \infty)\right)$. The space $W^{\sigma, 2}$ decreases with $\sigma$; we define the critical exponent $\beta^{*}$ of the family $W^{\sigma, 2}$ by

$$
\beta^{*}=2 \sup \left\{\sigma: \operatorname{dim} W^{\sigma, 2}=\infty\right\} .
$$

Clearly, the exponent $\beta^{*}$ is an invariant of the space $(M, d, \mu)$. For $M=\mathbb{R}^{n}$ and $\mu$ the Lebesgue measure, we have $\beta^{*}=2$.

To describe our results, assume that $\Phi_{1}(1)>0$ and consider the following three hypotheses for $\Phi_{2}$ :

$$
\begin{gathered}
\int_{0}^{\infty} s^{\alpha} \Phi_{2}(s) \frac{d s}{s}<\infty, \\
\int_{0}^{\infty} s^{\alpha+\beta} \Phi_{2}(s) \frac{d s}{s}<\infty,
\end{gathered}
$$




$$
\int_{0}^{\infty} s^{\alpha+\beta+\varepsilon} \Phi_{2}(s) \frac{d s}{s}<\infty,
$$

for some $\varepsilon>0$. We have proved the following.

Let $\left(H_{0}\right)$ hold. Then the measure of any metric ball $B(x, r):=\{y: d(x, y)<r\}$ in $M$ satisfies the estimates

$$
C^{-1} r^{\alpha} \leq \mu(B(x, r)) \leq C r^{\alpha},
$$

for all $x \in M$ and $r>0$, where $C$ is a positive constant (Theorem 3.2). In particular, this implies that $\alpha$ is intrinsically determined by $(M, d, \mu)$; moreover, $\alpha$ is the Hausdorff dimension of $M$.

The walk dimension $\beta$ satisfies the inequality $\beta \leq \beta^{*}$ where $\beta^{*}$ is the critical exponent of the family of Besov spaces defined above (Corollary 4.3 ). If, in addition, $(M, d)$ satisfies a certain chain condition (Definition 3.4), then $\beta \leq \alpha+1$ (Theorem $4.8(i i))$.

Let $\left(H_{1}\right)$ hold. Then the domain $\mathcal{E}(\mathcal{D})$ of the Dirichlet form $\mathcal{E}$ naturally associated with the heat kernel coincides with the Besov space $W^{\beta / 2,2}$ (Theorem 4.2). If $\alpha>\beta$, then the following embedding takes place:

$$
W^{\beta / 2,2}(M, \mu) \hookrightarrow L^{2^{*}}(M, \mu)
$$

where

$$
2^{*}:=\frac{2 \alpha}{\alpha-\beta},
$$

and the case $\alpha \leq \beta$ is also discussed (Theorem 4.11).

Let $\left(H_{2}\right)$ hold. Then $\beta=\beta^{*}$ (Theorem 4.6) and $\beta \geq 2$ (Theorem 4.8(i)).

Hence, under the hypothesis $\left(H_{2}\right)$ both parameters $\alpha$ and $\beta$ are uniquely determined by the underlying space. Note that under the hypothesis $\left(H_{0}\right)$ this is not the case, because, for example, both the Gauss-Weierstrass heat kernel (1.1) and the Cauchy-Poisson heat kernel (1.2) in $\mathbb{R}^{n}$ satisfy $\left(H_{0}\right)$, whereas for the former $\beta=2$ and for the latter $\beta=1$ (although $\alpha=n$ in both cases).

Let us mention that the characterization of the domain of the Dirichlet form on the Sierpiński gasket in terms of Besov spaces, as well as the relation $\beta=\beta^{*}$, was first obtained by A. Jonsson [18]. K. Pietruska-Pałuba obtained the similar conclusion for nested fractals [22]. Observe that the condition $\left(H_{1}\right)$ under which we prove the equivalence of $\mathcal{D}(\mathcal{E})$ and $W^{\beta / 2,2}$ is optimal. Indeed, for the heat kernel generated by the operator $-(-\Delta)^{\beta / 2}$ in $\mathbb{R}^{n}$ the function $\Phi_{2}$ is given by (1.6), and for this function the hypothesis $\left(H_{1}\right)$ breaks just on the borderline, because

$$
\int_{0}^{\infty} \frac{s^{\alpha+\beta}}{\left(1+s^{2}\right)^{\frac{\alpha+\beta}{2}}} \frac{d s}{s}=\infty .
$$

On the other hand, the domain of the energy form associated with the operator $-(-\Delta)^{\beta / 2}$ is known to be another Besov space Lip $(\beta / 2,2,2)$, which is smaller than $\operatorname{Lip}(\beta / 2,2, \infty)=W^{\beta / 2,2}$ (see [2], [23], [25]).

M. Barlow studied in [3] heat kernels on geodesic metric spaces, assuming that a heat kernel $p_{t}$ is the transition density of a diffusion process and that it satisfies (1.5) with functions $\Phi_{1}, \Phi_{2}$ of the type (1.7). Under these assumptions, he claimed that the walk dimension $\beta$ of $p_{t}$ is uniquely determined by the intrinsic properties of the underlying space (see [3, Theorem 3.21]). Under the additional assumption 
that $M$ is a subset of $\mathbb{R}^{n}$, this claim was proved in 23 . Our contribution is that we prove the uniqueness of $\beta$ for a general metric measure space $(M, d)$ and under a rather mild and nearly optimal condition $\left(H_{2}\right)$ on the heat kernel (nor do we assume existence of a diffusion process associated with $p_{t}$ ).

The relationship between the parameters $\alpha$ and $\beta$, mentioned above, is summarized in Corollary 4.9 as follows: If $(M, d)$ satisfies the chain condition, $\Phi_{1}(1)>0$ and, for some $\varepsilon>0$,

$$
\int_{0}^{\infty} s^{2 \alpha+\varepsilon} \Phi_{2}(s) d s<\infty
$$

then

$$
2 \leq \beta \leq \alpha+1 .
$$

The inequalities (1.11) were stated by Barlow in [3, Theorem 3.20], under the same set of assumptions as [3] Theorem 3.21]. Under somewhat more restrictive hypotheses, A. Stós [25] proved that $2 \leq \beta \leq \alpha+2$. In the setting of random walks in graphs, (1.11) was proved in [26] and [14], using techniques that are not available for general metric spaces. Our contribution is that we prove (1.11) under the hypothesis (1.10), which seems to be nearly optimal.

Barlow proved in [4] that for every pair $\alpha, \beta$ satisfying (1.11) there exists a random walk satisfying a discrete time version of (1.5) with these parameters. There is no doubt that the same is true for continuous time heat kernels. Hence, (1.11) is the only restriction on $\alpha$ and $\beta$.

In Section 5 we apply the embedding results to treat the following semilinear elliptic equation on $M$ :

$$
-\mathcal{L} u+f(x, u)=g(x),
$$

where $\mathcal{L}$ is the generator of the semigroup $T_{t}$ (the equation (1.12) arises for example when investigating the potential $u$ in porous or other irregular domains). We prove the existence and uniqueness results for weak solutions of (1.12), which in particular imply that, for all $q \geq p \geq 2^{*}$, the equation

$$
-\mathcal{L} u+|u|^{q-2} u=g
$$

has a unique weak solution $u \in \mathcal{D}(\mathcal{E}) \cap L^{p} \cap L^{q}$, for any $g \in L^{p^{\prime}}$, where $p^{\prime}=\frac{p}{p-1}$ (Theorem 5.4). The existence result relies on the compact embedding of the space $\mathcal{D}(\mathcal{E})$ into $L_{\text {loc }}^{2}$, which is true without any additional assumptions about $\Phi_{1}$ and $\Phi_{2}$ (Theorem 4.12).

Note that the classical existence results for equation (1.12) in $\mathbb{R}^{n}, n>2$, depend on the critical parameter $2^{*}=\frac{2 n}{n-2}$ (see [19]), which matches (1.9) since $\alpha=n$ and $\beta=2$.

Notation. The letters $C, c$ are used to denote positive constants whose values are unimportant but depend only on the hypotheses. The values of $C, c$ may be different at different occurrences.

For two non-negative functions $f(s)$ and $g(s)$ defined on a set $S$, we write

$$
f(s) \simeq g(s)
$$

if there is a constant $c$ such that for all $s \in S$,

$$
c^{-1} g(s) \leq f(s) \leq c g(s) .
$$




\section{Some EXAmples}

Let $l \geq 3$ be an integer and let $M_{0}=[0,1]^{n}(n \geq 2)$. We divide $M_{0}$ into $l^{n}$ equal subcubes. Remove a symmetric pattern of subcubes from $M_{0}$, and denote by $M_{1}$ what remains. Repeat the same procedure for each subcube in $M_{1}$ : divide each subcube into $l^{n}$ equal parts and remove the same pattern from each subcube, and denote by $M_{2}$ what remains. Continuing this way infinitely, we obtain a sequence of sets $\left\{M_{k}\right\}$. Set

$$
\widetilde{M}=\bigcap_{k=0}^{\infty} M_{k}
$$

and define

$$
M=\bigcup_{k=0}^{\infty} l^{k} \widetilde{M},
$$

where we write $a K=\{a x: x \in K\}$ for a real number $a$ and a set $K$.

The set $M$ is called an unbounded generalized Sierpinski carpet (cf. [5]); see Figure 1, which corresponds to the case $n=2$ and $l=3$.

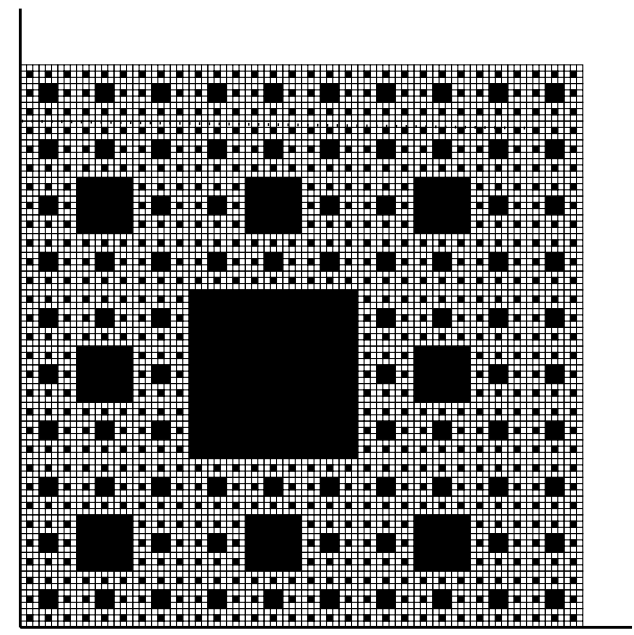

Figure 1. Generalized Sierpiński carpet

The distance $d$ on $M$ is set to be the Euclidean distance, and the measure $\mu$ is the Hausdorff measure of dimension $\alpha$, where $\alpha$ is the Hausdorff dimension of $M$. For any generalized Sierpiński carpet, there exists a heat kernel satisfying the following estimate:

$$
p_{t}(x, y) \simeq \frac{1}{t^{\alpha / \beta}} \exp \left(-c\left(\frac{d(x, y)^{\beta}}{t}\right)^{\frac{1}{\beta-1}}\right),
$$

which is a particular case of (1.5) with the functions $\Phi_{1}, \Phi_{2}$ of the form

$$
\Phi(s)=c \exp \left(-C s^{\frac{\beta}{\beta-1}}\right)
$$

(see [5]). There are also plenty of other fractals such that (2.1) holds; see, for example, [3], [11]. 
See also [13], [14, 20] for the heat kernel estimates in the setting of graphs or manifolds.

\section{Volume OF BALls}

Definition 3.1. We say that a heat kernel $p_{t}$ on a metric measure space $(M, d, \mu)$ satisfies the hypothesis $\left(H_{0}\right)$ if $p_{t}$ satisfies the estimate 1.5 with some positive constants $\alpha, \beta$ and non-negative decreasing functions $\Phi_{1}$ and $\Phi_{2}$ such that $\Phi_{1}(1)>0$ and

$$
\int_{0}^{\infty} s^{\alpha} \Phi_{2}(s) \frac{d s}{s}<\infty .
$$

Denote by $B(x, r)$ the metric ball in $M$ of radius $r$ centered at the point $x \in M$; that is,

$$
B(x, r):=\{y \in M: d(x, y)<r\} .
$$

Theorem 3.2. If a heat kernel $p_{t}$ satisfies the hypothesis $\left(H_{0}\right)$, then for all $x \in M$ and $r>0$,

$$
C^{-1} r^{\alpha} \leq \mu(B(x, r)) \leq C r^{\alpha} .
$$

Remark. The condition (3.1) is very mild. Indeed, if (3.2) is given, then (3.1) means that the upper bound function

$$
y \mapsto t^{-\alpha / \beta} \Phi_{2}\left(\frac{d(x, y)}{t^{1 / \beta}}\right)
$$

has a bounded integral over $M$ uniformly in $x$ and $t$, which resembles the normalization property of the heat kernel (cf. (3.7) below).

Note also that the estimate (3.2) can be true only for a single value of $\alpha$, which is called the exponent of the volume growth and is determined by intrinsic properties of the space $(M, d, \mu)$. Hence, under the hypothesis $\left(H_{0}\right)$ the value of the parameter $\alpha$ in (1.5) is an invariant of the underlying space.

Proof. Fix $x \in M$ and prove first the upper bound

$$
\mu(B(x, r)) \leq C r^{\alpha},
$$

for all $r>0$. Indeed, for any $t>0$, we have

$$
\int_{B(x, r)} p_{t}(x, y) d \mu(y) \leq \int_{M} p_{t}(x, y) d \mu(y)=1,
$$

whence

$$
\mu(B(x, r)) \leq\left(\inf _{y \in B(x, r)} p_{t}(x, y)\right)^{-1} .
$$

Taking $t=r^{\beta}$ and applying the lower bound in (1.5), we obtain

$$
\inf _{y \in B(x, r)} p_{t}(x, y) \geq \frac{1}{t^{\alpha / \beta}} \Phi_{1}(1)=c r^{-\alpha},
$$

whence (3.3) follows. An obvious modification of this argument allows us to replace the hypothesis $\Phi_{1}(1)>0$ by $\Phi_{1}\left(s_{0}\right)>0$ for some $s_{0}>0$.

Let us prove the opposite inequality

$$
\mu(B(x, r)) \geq c r^{\alpha} .
$$


We first show that the upper bounds in (1.5) and (3.3) imply that

$$
\int_{M \backslash B(x, r)} p_{t}(x, y) d \mu(y) \leq \frac{1}{2}, \quad \text { for all } t \leq \varepsilon r^{\beta},
$$

provided $\varepsilon>0$ is sufficiently small. Setting $r_{k}=2^{k} r$ and using the monotonicity of $\Phi_{2}$ and (3.3), we obtain

$$
\begin{aligned}
\int_{M \backslash B(x, r)} p_{t}(x, y) d \mu(y) & \leq \int_{M \backslash B(x, r)} t^{-\alpha / \beta} \Phi_{2}\left(\frac{d(x, y)}{t^{1 / \beta}}\right) d \mu(y) \\
& \leq \sum_{k=0}^{\infty} \int_{B\left(x, r_{k+1}\right) \backslash B\left(x, r_{k}\right)} t^{-\alpha / \beta} \Phi_{2}\left(\frac{r_{k}}{t^{1 / \beta}}\right) d \mu(y) \\
& \leq C \sum_{k=0}^{\infty} r_{k}^{\alpha} t^{-\alpha / \beta} \Phi_{2}\left(\frac{r_{k}}{t^{1 / \beta}}\right) \\
& =C \sum_{k=0}^{\infty}\left(\frac{2^{k} r}{t^{1 / \beta}}\right)^{\alpha} \Phi_{2}\left(\frac{2^{k} r}{t^{1 / \beta}}\right) \\
& \leq C \int_{\frac{1}{2} r / t^{1 / \beta}}^{\infty} s^{\alpha} \Phi_{2}(s) \frac{d s}{s} .
\end{aligned}
$$

The last line in (3.7) is proved as follows: setting $t_{k}=2^{k} r / t^{1 / \beta}$ for $k \geq-1$ and using the monotonicity of $\Phi_{2}$, we obtain

$$
\begin{aligned}
\int_{\frac{1}{2} r / t^{1 / \beta}}^{\infty} s^{\alpha} \Phi_{2}(s) \frac{d s}{s} & =\sum_{k=0}^{\infty} \int_{t_{k-1}}^{t_{k}} s^{\alpha-1} \Phi_{2}(s) d s \\
& \geq \frac{1}{\alpha} \sum_{k=0}^{\infty} \Phi_{2}\left(t_{k}\right)\left(t_{k}^{\alpha}-t_{k-1}^{\alpha}\right) \\
& =c \sum_{k=0}^{\infty} \Phi_{2}\left(t_{k}\right) t_{k}^{\alpha} .
\end{aligned}
$$

Since by (3.1) the integral in (3.7) is convergent, its value can be made arbitrarily small provided $r^{\beta} / t$ is large enough, whence (3.6) follows.

From the normalization property and (3.6), we conclude that for such $r$ and $t$,

$$
\int_{B(x, r)} p_{t}(x, y) d \mu(y) \geq \frac{1}{2},
$$

whence

$$
\mu(B(x, r)) \geq \frac{1}{2}\left(\sup _{y \in B(x, r)} p_{t}(x, y)\right)^{-1} .
$$

Finally, choosing $t:=\varepsilon r^{\beta}$ and using the upper bound

$$
p_{t}(x, y) \leq t^{-\alpha / \beta} \Phi_{2}(0)=C r^{-\alpha},
$$

we obtain (3.5).

Note that the method we have used in the proof of Theorem[3.2 is close to [14].

Corollary 3.3. Let $(M, d, \mu)$ be a metric measure space, and $p_{t}$ be a heat kernel on $M$ satisfying (1.5). If $\Phi_{1}(1)>0$, then $\operatorname{diam}(M)=\infty$. 
Proof. Let us show that the upper bound in (1.5) implies $\mu(M)=\infty$. Indeed, fix a point $x_{0} \in M$ and observe that the family of functions $u_{t}(x)=p_{t}\left(x, x_{0}\right)$ satisfies the following two conditions:

$$
\left\|u_{t}\right\|_{1}=1
$$

and

$$
\left\|u_{t}\right\|_{\infty} \leq C t^{-\alpha / \beta} \rightarrow 0 \text { as } t \rightarrow \infty
$$

where $\|\cdot\|_{q}$ stands for the $L^{q}(M, \mu)$ norm. Hence, we obtain

$$
\mu(M) \geq \frac{\left\|u_{t}\right\|_{1}}{\left\|u_{t}\right\|_{\infty}} \rightarrow \infty \quad \text { as } t \rightarrow \infty
$$

that is, $\mu(M)=\infty$.

On the other hand, the first part of the proof of Theorem 3.2, based solely on the hypothesis $\Phi_{1}(1)>0$, says that the measure of any ball is finite. Hence, $M$ is not contained in any ball, which ends the proof.

Under a mild additional hypothesis about the metric space $(M, d)$, we will obtain a self-improved lower bound for the heat kernel.

Definition 3.4. We say that a metric space $(M, d)$ satisfies the chain condition if there exists a (large) constant $C$ such that for any two points $x, y \in M$ and for any positive integer $n$ there exists a sequence $\left\{x_{i}\right\}_{i=0}^{n}$ of points in $M$ such that $x_{0}=x$, $x_{n}=y$, and

$$
d\left(x_{i}, x_{i+1}\right) \leq C \frac{d(x, y)}{n}, \quad \text { for all } i=0,1, \ldots, n-1 .
$$

The sequence $\left\{x_{i}\right\}_{i=0}^{n}$ is referred to as a chain connecting $x$ and $y$.

For example, the chain condition is satisfied if $(M, d)$ is a length space, that is, if the distance $d(x, y)$ is defined as the infimum of the length of all continuous curves connecting $x$ and $y$, with a proper definition of length. On the other hand, the chain condition is not satisfied if $M$ is a locally finite graph, and $d$ is the graph distance.

Corollary 3.5. Under the hypotheses of Theorem 3.2, assume in addition that $\beta>1$ and that $(M, d)$ satisfies the chain condition. Then the following inequality holds, for all $x, y \in M$ and $t>0$ :

$$
p_{t}(x, y) \geq \frac{c}{t^{\alpha / \beta}} \exp \left(-C\left(\frac{d(x, y)^{\beta}}{t}\right)^{\frac{1}{\beta-1}}\right) .
$$

Remark. The assumption $\beta>1$ is not very restrictive. In fact, as we will see in Section 4.5 (1.5) implies $\beta \geq 2$ provided $\Phi_{2}$ satisfies a condition slightly stronger than (3.1). On the other hand, the condition $\beta>1$ does not follow from the hypotheses of Theorem 3.2, because the Cauchy-Poisson heat kernel (1.2) satisfies them whereas for this heat kernel $\beta=1$.

Finally, let us observe that (3.10) matches the lower bound in (2.1). 
Proof. By iterating the semigroup identity, we obtain, for any positive integer $n$ and real $r>0$,

$$
\begin{aligned}
p_{t}(x, y) & =\int \ldots \int p_{\frac{t}{n}}\left(x, z_{1}\right) p_{\frac{t}{n}}\left(z_{1}, z_{2}\right) \ldots p_{\frac{t}{n}}\left(z_{n-1}, y\right) d \mu\left(z_{1}\right) \ldots d \mu\left(z_{n-1}\right) \\
(3.11) & \geq \int_{B\left(x_{1}, r\right)} \ldots \int_{B\left(x_{n-1}, r\right)} p_{\frac{t}{n}}\left(x, z_{1}\right) p_{\frac{t}{n}}\left(z_{1}, z_{2}\right) \ldots p_{\frac{t}{n}}\left(z_{n-1}, y\right) d \mu\left(z_{n-1}\right) \ldots d \mu\left(z_{1}\right),
\end{aligned}
$$

where $\left\{x_{i}\right\}_{i=0}^{n}$ is a chain connecting $x$ and $y$.

Denote for simplicity $z_{0}=x$ and $z_{n}=y$. Since $z_{i} \in B\left(x_{i}, r\right), 0 \leq i \leq n-1$, we obtain by the triangle inequality and (3.9),

$$
d\left(z_{i}, z_{i+1}\right) \leq d\left(x_{i}, x_{i+1}\right)+2 r \leq C \frac{d(x, y)}{n}+2 r .
$$

Choosing

$$
r=\frac{d(x, y)}{n}
$$

and using the lower bound in 1.5), we obtain

$$
p_{\frac{t}{n}}\left(z_{i}, z_{i+1}\right) \geq \frac{1}{(t / n)^{\alpha / \beta}} \Phi_{1}\left(\frac{d\left(z_{i}, z_{i+1}\right)}{(t / n)^{1 / \beta}}\right) \geq \frac{n^{\alpha / \beta}}{t^{\alpha / \beta}} \Phi_{1}\left(\frac{d(x, y)}{\left(c t n^{\beta-1}\right)^{1 / \beta}}\right),
$$

where $c=(C+2)^{-\beta}$.

If $d(x, y)^{\beta} \leq t$, then (3.10) follows immediately from (1.5). Assume in the sequel that $d(x, y)^{\beta}>t$. Choosing $n$ to be the least positive integer satisfying the inequality

we obtain from (3.13),

$$
c t n^{\beta-1} \geq d(x, y)^{\beta}
$$

$$
p_{\frac{t}{n}}\left(z_{i}, z_{i+1}\right) \geq \frac{n^{\alpha / \beta}}{t^{\alpha / \beta}} \Phi_{1}(1) .
$$

Hence, (3.11), (3.2), and (3.12) imply

$$
\begin{aligned}
p_{t}(x, y) & \geq\left(c \frac{n^{\alpha / \beta}}{t^{\alpha / \beta}}\right)^{n} \prod_{i=1}^{n-1} \mu\left(B\left(x_{i}, r\right)\right) \\
& \geq\left(c \frac{n^{a / \beta}}{t^{a / \beta}}\right)^{n}\left(c r^{\alpha}\right)^{n-1} \\
& =c\left(\frac{n}{t}\right)^{\alpha / \beta}\left(c \frac{d(x, y)^{\beta}}{n^{\beta-1} t}\right)^{(n-1) \alpha / \beta} .
\end{aligned}
$$

Since $n^{\beta-1} \simeq d(x, y)^{\beta} / t$, we see that

$$
\left(c \frac{d(x, y)^{\beta}}{n^{\beta-1} t}\right)^{(n-1) \alpha / \beta} \geq \varepsilon^{n}
$$

for some (small) positive constant $\varepsilon$, which together with (3.14) yields

$$
p_{t}(x, y) \geq c\left(\frac{n}{t}\right)^{\alpha / \beta} \varepsilon^{n} \geq \frac{c}{t^{\alpha / \beta}} \exp (-C n) \geq \frac{c}{t^{\alpha / \beta}} \exp \left(-C\left(\frac{d(x, y)^{\beta}}{t}\right)^{\frac{1}{\beta-1}}\right) \text {. }
$$




\section{BESOV SPACES}

Let $(M, d, \mu)$ be a metric measure space. For any $q \in[1,+\infty]$, set $L^{q}=L^{q}(M, \mu)$ and

$$
\|u\|_{q}=\|u\|_{L^{q}(M, \mu)} .
$$

For any $\sigma>0$, define a non-negative functional $W_{\sigma}(u)$ on measurable functions on $M$ by

$$
W_{\sigma}(u):=\sup _{0<r<1} r^{-2 \sigma} \int_{M}\left[\frac{1}{\mu(B(x, r))} \int_{B(x, r)}|u(y)-u(x)|^{2} d \mu(y)\right] d \mu(x) .
$$

In particular, if the condition (3.2) is satisfied, then for any $\beta>0$,

$$
W_{\beta / 2}(u) \simeq \sup _{0<r<1} r^{-\alpha-\beta} \int_{M}\left[\int_{B(x, r)}|u(y)-u(x)|^{2} d \mu(y)\right] d \mu(x) .
$$

Define the space $W^{\sigma, 2}$ as follows:

$$
W^{\sigma, 2}=W^{\sigma, 2}(M, d, \mu):=\left\{u \in L^{2}: W_{\sigma}(u)<\infty\right\} .
$$

It is easy to see that $W_{\sigma}^{1 / 2}$ is a semi-norm in $W^{\sigma, 2}$, and $W^{\sigma, 2}$ is a Banach space with the norm

$$
\|u\|_{\sigma, 2}:=\|u\|_{2}+W_{\sigma}(u)^{1 / 2}
$$

(see for example [18]). The space $W^{\sigma, 2}$ is one of the family of Besov spaces, and it is similar to the space that was denoted by $\operatorname{Lip}(\sigma, 2, \infty)$ in $[18$.

4.1. The Laplace operator. Define by 1.3 the semigroup $\left\{T_{t}\right\}_{t>0}$ in $L^{2}$, and consider its infinitesimal generator $\mathcal{L}$ defined by

$$
\mathcal{L} u:=\lim _{t \rightarrow 0} \frac{T_{t} u-u}{t},
$$

where the limit is understood in the $L^{2}$-norm. It is natural to refer to $\mathcal{L}$ as the Laplace operator of the heat kernel $p_{t}$. The domain $\operatorname{dom}(\mathcal{L})$ of the operator $\mathcal{L}$ is the space of functions $u \in L^{2}$ for which the limit in (4.3) exists. Since $\left\{T_{t}\right\}$ is a strongly continuous contraction semigroup in $L^{2}$, by the Hille-Yosida theorem $\operatorname{dom}(\mathcal{L})$ is dense in $L^{2}$; furthermore, $\mathcal{L}$ is a selfadjoint, non-positive-definite operator, which follows from the fact that $T_{t}$ is selfadjoint and contractive (see for example [8, 12], [16], or [29, Theorem 1, p. 237]).

4.2. The Dirichlet form. For any $t>0$, define the quadratic form $\mathcal{E}_{t}$ on $L^{2}$ by

$$
\mathcal{E}_{t}[u]:=\left(\frac{u-T_{t} u}{t}, u\right),
$$

where $($,$) is the inner product in L^{2}$. An easy computation shows that $\mathcal{E}_{t}$ can be equivalently defined by

$$
\mathcal{E}_{t}[u]=\frac{1}{2 t} \int_{M} \int_{M}(u(x)-u(y))^{2} p_{t}(x, y) d \mu(y) d \mu(x) .
$$

In terms of the spectral resolution $\left\{E_{\lambda}\right\}$ of the operator $-\mathcal{L}, \mathcal{E}_{t}$ can be expressed as follows:

$$
\mathcal{E}_{t}[u]=\int_{0}^{\infty} \frac{1-e^{-t \lambda}}{t} d\left\|E_{\lambda} u\right\|_{2}^{2},
$$

which implies that $\mathcal{E}_{t}[u]$ is decreasing in $t$ (see also [7]). 
Let us define a quadratic form $\mathcal{E}$ by

$$
\mathcal{E}[u]:=\lim _{t \rightarrow 0+} \mathcal{E}_{t}[u]
$$

(where the limit may be $+\infty$ since $\mathcal{E}[u] \geq \mathcal{E}_{t}[u]$ ) and its domain $\mathcal{D}(\mathcal{E})$ by

$$
\mathcal{D}(\mathcal{E}):=\left\{u \in L^{2}: \mathcal{E}[u]<\infty\right\} .
$$

By a standard procedure, the quadratic form $\mathcal{E}[u]$ extends to the bilinear form $\mathcal{E}(u, v)$.

From (4.5) it follows that $\mathcal{E}$ is non-negative definite. The domain $\mathcal{D}(\mathcal{E})$ is dense in $L^{2}$ because $\mathcal{D}(\mathcal{E})$ contains $\operatorname{dom}(\mathcal{L})$. Moreover, the form $\mathcal{E}$ is closed; that is, the space $\mathcal{D}(\mathcal{E})$ is a Hilbert space with the inner product

$$
[u, v]:=(u, v)+\mathcal{E}(u, v) .
$$

Clearly (4.3) and (4.4) imply that

$$
\mathcal{E}(u, v)=(-\mathcal{L} u, v),
$$

for all $u \in \operatorname{dom}(\mathcal{L})$ and $v \in \mathcal{D}(\mathcal{E})$.

It is easy to see from (1.3) and the definition of a heat kernel that the semigroup $\left\{T_{t}\right\}$ is Markovian, that is, $0 \leq u \leq 1$ implies $0 \leq T_{t} u \leq 1$. By [12, Theorem 1.4.1], the form $\mathcal{E}$ satisfies the Markov property, that is, $u \in \mathcal{D}(\mathcal{E})$ implies $v:=$ $\min \left(u_{+}, 1\right) \in \mathcal{D}(\mathcal{E})$ and $\mathcal{E}[v] \leq \mathcal{E}[u]$. Hence, $\mathcal{E}$ is a Dirichlet form.

\subsection{Besov space as the domain of the Dirichlet form.}

Definition 4.1. We say that a heat kernel $p_{t}$ on a metric measure space $(M, d, \mu)$ satisfies the hypothesis $\left(H_{1}\right)$ if $p_{t}$ satisfies the estimate (1.5) with some positive constants $\alpha, \beta$ and non-negative decreasing functions $\Phi_{1}$ and $\Phi_{2}$ such that $\Phi_{1}(1)>0$ and

$$
\int_{0}^{\infty} s^{\alpha+\beta} \Phi_{2}(s) \frac{d s}{s}<\infty .
$$

The following theorem identifies the domain $\mathcal{D}(\mathcal{E})$ of the Dirichlet form with a Besov space.

Theorem 4.2. Let $p_{t}$ be a heat kernel on $(M, d, \mu)$ satisfying the hypothesis $\left(H_{1}\right)$, and let $\mathcal{E}$ be the Dirichlet form defined by (4.5) and (4.6). Then

$$
\mathcal{D}(\mathcal{E})=W^{\beta / 2,2}
$$

and for any $u \in \mathcal{D}(\mathcal{E})$,

$$
\mathcal{E}[u] \simeq W_{\beta / 2}(u) .
$$

Proof. Since the expressions $\mathcal{E}[u]$ and $W_{\beta / 2}(u)$ are defined for all $u \in L^{2}$, it suffices to show that (4.10) holds for all $u \in L^{2}$ (allowing infinite values for both sides). Note that the hypotheses of Theorem 3.2 hold, so that we have the estimate (3.2) of the volumes of balls.

We first prove that

$$
\mathcal{E}[u] \geq c W_{\beta / 2}(u)
$$


using the approach of [23. Using the lower bound in (1.5) and the monotonicity of $\Phi_{1}$, we obtain from (4.5) that for any $r>0$ and $t=r^{\beta}$,

$$
\begin{aligned}
\mathcal{E}[u] & \geq \frac{1}{2 t} \int_{M} \int_{B(x, r)}(u(x)-u(y))^{2} p_{t}(x, y) d \mu(y) d \mu(x) \\
& \geq \frac{1}{2}\left(\frac{1}{t}\right)^{\alpha / \beta+1} \Phi_{1}\left(\frac{r}{t^{1 / \beta}}\right) \int_{M} \int_{B(x, r)}(u(x)-u(y))^{2} d \mu(y) d \mu(x) \\
& =\frac{\Phi_{1}(1)}{2} r^{-(\alpha+\beta)} \int_{M} \int_{B(x, r)}(u(x)-u(y))^{2} d \mu(y) d \mu(x) .
\end{aligned}
$$

Together with (4.2), this implies (4.11).

Let us now prove the opposite inequality, that is,

$$
\mathcal{E}[u] \leq C W_{\beta / 2}(u) .
$$

For any $t>0$ we have

$$
\mathcal{E}_{t}[u]=\frac{1}{2 t} \int_{M} \int_{M}(u(x)-u(y))^{2} p_{t}(x, y) d \mu(y) d \mu(x)=A(t)+B(t)
$$

where

$$
\begin{aligned}
& A(t)=\frac{1}{2 t} \int_{M} \int_{M \backslash B(x, 1)}(u(x)-u(y))^{2} p_{t}(x, y) d \mu(y) d \mu(x), \\
& B(t)=\frac{1}{2 t} \int_{M} \int_{B(x, 1)}(u(x)-u(y))^{2} p_{t}(x, y) d \mu(y) d \mu(x) .
\end{aligned}
$$

To estimate $A(t)$ let us observe that by (3.7),

$$
\int_{M \backslash B(x, 1)} p_{t}(x, y) d \mu(y) \leq C \int_{\frac{1}{2 t^{1 / \beta}}}^{\infty} s^{\alpha} \Phi_{2}(s) \frac{d s}{s} \leq C t \int_{\frac{1}{2 t^{1 / \beta}}}^{\infty} s^{\alpha+\beta} \Phi_{2}(s) \frac{d s}{s},
$$

whence by (4.9),

$$
\int_{M \backslash B(x, 1)} p_{t}(x, y) d \mu(y)=o(t) \quad \text { as } t \rightarrow 0+\text { uniformly in } x \in M .
$$

Therefore, applying the elementary inequality $(a-b)^{2} \leq 2\left(a^{2}+b^{2}\right)$ in (4.13) and using (4.16), we obtain that for small enough $t>0$,

$$
\begin{aligned}
A(t) & \leq \frac{1}{t} \int_{M} \int_{M \backslash B(x, 1)}\left(u(x)^{2}+u(y)^{2}\right) p_{t}(x, y) d \mu(y) d \mu(x) \\
& =\frac{2}{t} \int_{M} u(x)^{2}\left(\int_{M \backslash B(x, 1)} p_{t}(x, y) d \mu(y)\right) d \mu(x) \\
& =o(1)\|u\|_{2}^{2},
\end{aligned}
$$

whence

$$
\lim _{t \rightarrow 0+} A(t)=0 .
$$


The quantity $B(t)$ is estimated as follows using (1.5), (4.2), and (4.9):

$$
\begin{aligned}
B(t) & =\frac{1}{2 t} \sum_{k=1}^{\infty} \int_{M} \int_{B\left(x, 2^{-(k-1)}\right) \backslash B\left(x, 2^{-k}\right)}(u(x)-u(y))^{2} p_{t}(x, y) d \mu(y) d \mu(x) \\
& \leq \frac{1}{2} \sum_{k=1}^{\infty}\left(\frac{1}{t}\right)^{\alpha / \beta+1} \Phi_{2}\left(\frac{2^{-k}}{t^{1 / \beta}}\right) \int_{M} \int_{B\left(x, 2^{-(k-1)}\right)}(u(x)-u(y))^{2} d \mu(y) d \mu(x) \\
& \leq C W_{\beta / 2}(u) \sum_{k=1}^{\infty}\left(\frac{2^{-k}}{t^{1 / \beta}}\right)^{\alpha+\beta} \Phi_{2}\left(\frac{2^{-k}}{t^{1 / \beta}}\right) \\
& \leq C W_{\beta / 2}(u) \int_{0}^{\infty} s^{\alpha+\beta} \Phi_{2}(s) \frac{d s}{s} \\
(4.18) & \leq C W_{\beta / 2}(u) .
\end{aligned}
$$

It follows from (4.12), (4.17) and 4.18) that

$$
\mathcal{E}[u]=\lim _{t \rightarrow 0+} \mathcal{E}_{t}[u] \leq C W_{\beta / 2}(u),
$$

which finishes the proof.

4.4. Intrinsic characterization of the walk dimension. As one can see from the above proof of Theorem 4.2 the inclusion $\mathcal{D}(\mathcal{E}) \subset W^{\beta / 2,2}$ requires only the lower estimates in (1.5) and (3.2) (and the opposite inclusion requires only the upper estimates in (1.5) and (3.2)). This observation allows us to prove the following statement.

Corollary 4.3. If a heat kernel satisfies the hypotheses $\left(H_{0}\right)$ (cf. Theorem [3.2), then $\operatorname{dim} W^{\beta / 2,2}=\infty$.

Proof. Indeed, by Theorem 3.2 the hypothesis $\left(H_{0}\right)$ implies (3.2), and by the above remark we obtain $\mathcal{D}(\mathcal{E}) \subset W^{\beta / 2,2}$. On the other hand, $\mathcal{D}(\mathcal{E})$ is always dense in $L^{2}$, whereas by Corollary 3.3 we have $\operatorname{dim} L^{2}=\infty$, whence the claim follows.

Definition 4.4. Let us set

$$
\beta^{*}:=2 \sup \left\{\sigma: \operatorname{dim} W^{\sigma, 2}=\infty\right\}
$$

and call $\beta^{*}$ the critical exponent of the family $W^{\sigma, 2}$ of the Besov spaces in $(M, d, \mu)$.

Note that the value of $\beta^{*}$ is an intrinsic property of the space $(M, d, \mu)$, which is defined independently of any heat kernel. For example, for $\mathbb{R}^{n}$ we have $\beta^{*}=2$. In terms of the critical exponent, Corollary 4.3 can be stated as follows: if a heat kernel satisfies $\left(H_{0}\right)$, then

$$
\beta \leq \beta^{*},
$$

where $\beta$ is the walk dimension of the heat kernel. In general, it may happen that $\beta<\beta^{*}$, as one can see from the example of the Cauchy-Poisson heat kernel in $\mathbb{R}^{n}$ where $\beta=1$ and $\beta^{*}=2$. A theorem below provides conditions to ensure $\beta=\beta^{*}$ (see also [17]).

Definition 4.5. We say that a heat kernel $p_{t}$ on a metric measure space $(M, d, \mu)$ satisfies the hypothesis $\left(H_{2}\right)$ if $p_{t}$ satisfies the estimate (1.5) with some positive 
constants $\alpha, \beta$ and non-negative decreasing functions $\Phi_{1}$ and $\Phi_{2}$ such that $\Phi_{1}(1)>0$ and, for some $\varepsilon>0$,

$$
\int_{0}^{\infty} s^{\alpha+\beta+\varepsilon} \Phi_{2}(s) \frac{d s}{s}<\infty .
$$

Note that the hypothesis $\left(H_{2}\right)$ is stronger than $\left(H_{1}\right)$, and $\left(H_{1}\right)$ is stronger than $\left(H_{0}\right)$, but still $\left(H_{2}\right)$ is satisfied in most applications. For example, every function of the form

$$
\Phi_{2}(s)=\exp \left(-c s^{\gamma}\right)
$$

satisfies $\left(H_{2}\right)$ provided $c>0$ and $\gamma>0$.

Theorem 4.6. If $p_{t}$ is a heat kernel satisfying the hypothesis $\left(H_{2}\right)$, then for any $\sigma>\beta / 2$ the space $W^{\sigma, 2}$ contains only zero. Consequently, $\beta=\beta^{*}$.

Remark. As follows from Corollary 4.3, the conclusion of Theorem 4.6 remains true if $\beta$ in (4.20) is replaced by $\beta^{*}$, which makes the hypotheses of Theorem 4.6 independent of $\beta$.

Proof. Let us explain why $\beta=\beta^{*}$ follows from the first claim. Indeed, $\operatorname{dim} W^{\sigma, 2}=0$ implies that $\sigma \geq \beta^{*} / 2$, and since this is true for any $\sigma>\beta / 2$, we obtain $\beta \geq \beta^{*}$. Since the opposite inequality holds by Corollary 4.3 , we conclude that $\beta=\beta^{*}$.

The proof of the first claim is similar to that of Theorem 4.2 Fix $\sigma>\beta / 2$ and a function $u \in W^{\sigma, 2}$, and show that $\mathcal{E}[u]=0$. Note that if (4.20) holds for some $\varepsilon>0$, it also holds for a smaller value of $\varepsilon$. Thus we may assume $\varepsilon$ to be so small that

$$
2 \sigma-\beta-\varepsilon>0
$$

Again we use the decomposition $\mathcal{E}_{t}[u]=A(t)+B(t)$, where $A(t)$ and $B(t)$ are defined in (4.13) and (4.14). Similar to (4.18) but using $W_{\sigma}$ instead of $W_{\beta / 2}$, we estimate $B(t)$ and obtain by (4.1), 4.22), and (4.20),

$$
\begin{aligned}
& t^{-\varepsilon / \beta} B(t) \\
& \leq \frac{1}{2} \sum_{k=1}^{\infty}\left(\frac{1}{t}\right)^{(\alpha+\varepsilon) / \beta+1} \Phi_{2}\left(\frac{2^{-k}}{t^{1 / \beta}}\right) \int_{M} \int_{B\left(x, 2^{-(k-1)}\right)}(u(x)-u(y))^{2} d \mu(y) d \mu(x) \\
& \leq C W_{\sigma}(u) \sum_{k=1}^{\infty} 2^{-k(2 \sigma+\alpha)}\left(\frac{1}{t}\right)^{(\alpha+\varepsilon) / \beta+1} \Phi_{2}\left(\frac{2^{-k}}{t^{1 / \beta}}\right) \\
& =C W_{\sigma}(u) \sum_{k=1}^{\infty} 2^{-k(2 \sigma-\beta-\varepsilon)}\left(\frac{2^{-k}}{t^{1 / \beta}}\right)^{\alpha+\beta+\varepsilon} \Phi_{2}\left(\frac{2^{-k}}{t^{1 / \beta}}\right) \\
& \leq C W_{\sigma}(u) \int_{0}^{\infty} s^{\alpha+\beta+\varepsilon} \Phi_{2}(s) \frac{d s}{s} \\
& \leq C W_{\sigma}(u) .
\end{aligned}
$$

Together with (4.12) and (4.17) this yields

$$
\mathcal{E}_{t}[u] \leq A(t)+C t^{\varepsilon / \beta} W_{\sigma}(u) \rightarrow 0 \quad \text { as } t \rightarrow 0,
$$

whence

$$
\mathcal{E}[u]=\lim _{t \rightarrow 0} \mathcal{E}_{t}[u]=0 .
$$


Since $\mathcal{E}_{t}[u] \leq \mathcal{E}[u]$, this implies in return that $\mathcal{E}_{t}[u] \equiv 0$ for all $t>0$. On the other hand, it follows from (4.5) and the lower bound in (1.5) that

$$
\mathcal{E}_{t}[u] \geq \frac{1}{2 t^{\alpha / \beta+1}} \Phi_{1}(1) \iint_{\left\{x, y: d(x, y) \leq t^{1 / \beta}\right\}}(u(x)-u(y))^{2} d \mu(y) d \mu(x),
$$

which yields $u(x)=u(y)$ for $\mu$-almost all $x, y$ such that $d(x, y) \leq t^{1 / \beta}$. Since $t$ is arbitrary, we conclude that $u$ is a constant function. Since $\mu(M)=\infty$ (see Corollary 3.3), we obtain $u \equiv 0$.

Corollary 4.7. If a heat kernel $p_{t}$ satisfies $\left(H_{2}\right)$, then the values of the parameters $\alpha$ and $\beta$ in (1.5) are invariants of the space $(M, d, \mu)$. In particular, if there are two heat kernels $p_{t}^{(1)}$ and $p_{t}^{(2)}$ on $(M, d, \mu)$ both satisfying $\left(H_{2}\right)$ with parameters $\alpha_{1}, \beta_{1}$ and $\alpha_{2}, \beta_{2}$ respectively, then $\alpha_{1}=\alpha_{2}$ and $\beta_{1}=\beta_{2}$.

Proof. By Theorem 3.2, $\mu(B(x, r))$ satisfies (3.2), which uniquely determines $\alpha$ as the exponent of the volume growth of $(M, d, \mu)$. By Theorem 4.6 $\beta$ is uniquely determined as the critical exponent of the family of Besov spaces of $(M, d, \mu)$.

Remark. Although $\alpha_{1}=\alpha_{2}$ is true already under the hypothesis $\left(H_{0}\right)$, this hypothesis is not enough to ensure $\beta_{1}=\beta_{2}$. Indeed, $\mathbb{R}^{n}$ carries the Gauss-Weierstrass heat kernel $p_{t}^{(1)}$ given by (1.1) and the Cauchy-Poisson heat kernel $p_{t}^{(2)}$ given by (1.2), and for these heat kernels we have $\alpha_{1}=\alpha_{2}=n$ whereas $\beta_{1}=2 \neq 1=\beta_{2}$.

\subsection{Inequalities for the walk dimension.}

Theorem 4.8. Let $p_{t}$ be a heat kernel on a metric measure space $(M, d, \mu)$.

(i) If $p_{t}$ satisfies the hypothesis $\left(H_{2}\right)$ (cf. Theorem 4.6), then

$$
\beta \geq 2 \text {. }
$$

(ii) If $p_{t}$ satisfies the hypothesis $\left(H_{0}\right)$ (cf. Theorem 3.2) and the space $(M, d)$ satisfies the chain condition (cf. Definition 3.4), then

$$
\beta \leq \alpha+1 \text {. }
$$

Remark. Observe that the chain condition is essential for the inequality $\beta \leq \alpha+1$ to be true. Indeed, assume for a moment that the claim of Theorem 4.8(ii) holds without the chain condition, and consider a new metric $d_{\gamma}$ on $M$ given by $d_{\gamma}=$ $d^{1 / \gamma}$ where $\gamma>1$. It is easy to see that the heat kernel $p_{t}$ considered on the space $\left(M, d_{\gamma}, \mu\right)$ will satisfy (1.5) with parameters $\alpha \gamma$ and $\beta \gamma$ instead of $\alpha$ and $\beta$, respectively. Hence, Theorem 4.8(ii) would yield $\beta \gamma \leq \alpha \gamma+1$, which implies $\beta \leq \alpha$ because $\gamma$ may be taken arbitrarily large. However, there are spaces with $\beta>\alpha$, for example the classical Sierpiński gasket. Clearly, the metric $d_{\gamma}$ does not satisfy the chain condition; indeed, the inequality (3.9) implies

$$
d_{\gamma}\left(x_{i}, x_{i+1}\right) \leq C \frac{d_{\gamma}(x, y)}{n^{1 / \gamma}},
$$

which is not good enough. If in the inequality (3.9) we replace $n$ by $n^{1 / \gamma}$, then the proof will give $\beta \leq \alpha+\gamma$ instead of $\beta \leq \alpha+1$.

Corollary 4.9. Let a metric space $(M, d)$ satisfy the chain condition. Assume that a heat kernel $p_{t}$ on $(M, d, \mu)$ satisfies the estimate (1.5) with positive parameters 
$\alpha, \beta$ and non-negative decreasing functions $\Phi_{1}$ and $\Phi_{2}$ such that $\Phi_{1}(1)>0$ and, for some $\varepsilon>0$,

$$
\int_{0}^{\infty} s^{2 \alpha+1+\varepsilon} \Phi_{2}(s) \frac{d s}{s}<\infty
$$

Then

$$
2 \leq \beta \leq \alpha+1 .
$$

Proof of Corollary 4.9. Clearly, (4.26) implies $\left(H_{0}\right)$, and by Theorem 4.8 (ii) we obtain $\beta \leq \alpha+1$. Therefore, (4.26) implies $\left(H_{2}\right)$, and by Theorem $4.8(i)$ we obtain $\beta \geq 2$.

Proof of Theorem $4.8(i)$. Let us show that the space $W^{1,2}$ is non-trivial, which would imply by Theorem 4.6 that $\beta \geq 2$. Fix a ball $B\left(x_{0}, R\right)$ in $M$ and let $u(x)$ be the test function of this ball, that is,

$$
u(x)=\left(R-d\left(x, x_{0}\right)\right)_{+} .
$$

Since $\operatorname{diam}(M)=\infty$ (see Corollary 3.3), this function is non-constant for large enough $R$. Let us show that $W_{1}(u)<\infty$. Indeed, by (4.2), it suffices to prove that for some constant $C$ and for all $r \in(0,1)$,

$$
U(r):=r^{-2-\alpha} \int_{M}\left[\int_{B(x, r)}|u(x)-u(y)|^{2} d \mu(y)\right] d \mu(x)<C .
$$

Since the function $u$ vanishes outside the ball $B\left(x_{0}, R\right)$ and $r<1$, the exterior integration in (4.28) can be reduced to $B\left(x_{0}, R+1\right)$. Hence, using the obvious inequality

$$
|u(x)-u(y)| \leq d(x, y) \leq r
$$

we obtain

$$
\begin{aligned}
U(r) & =r^{-2-\alpha} \int_{B\left(x_{0}, R+1\right)}\left[\int_{B(x, r)}|u(y)-u(x)|^{2} d \mu(y)\right] d \mu(x) \\
& \leq r^{-2-\alpha} \int_{B\left(x_{0}, R+1\right)} r^{2} \mu(B(x, r)) d \mu(x) \\
& \leq C r^{-2-\alpha} \int_{B\left(x_{0}, R+1\right)} r^{2+\alpha} d \mu(x) \\
& =C \mu\left(B\left(x_{0}, R+1\right)\right),
\end{aligned}
$$

whence (4.28) follows.

We precede the proof of the second part of Theorem 4.8 by two lemmas.

Lemma 4.10. Let $\left\{x_{i}\right\}_{i=0}^{n}$ be a sequence of points in a metric space $(M, d)$ such that for some $\rho>0$ we have $d\left(x_{0}, x_{n}\right)>2 \rho$ and

$$
d\left(x_{i}, x_{i+1}\right)<\rho \text { for all } i=0,1, \ldots, n-1 .
$$

Then there exists a subsequence $\left\{x_{i_{k}}\right\}_{k=0}^{l}$ such that

(a) $0=i_{0}<i_{1}<\ldots<i_{l}=n$;

(b) $d\left(x_{i_{k}}, x_{i_{k+1}}\right)<5 \rho$ for all $k=0,1, \ldots, l-1$;

(c) $d\left(x_{i_{k}}, x_{i_{j}}\right) \geq 2 \rho$ for all distinct $k, j=0,1, \ldots, l$. 
The significance of conditions $(a),(b),(c)$ is that a sequence $\left\{x_{i_{k}}\right\}_{k=0}^{l}$ satisfying them gives rise to a chain of balls $B\left(x_{i_{k}}, 5 \rho\right)$ connecting the points $x_{0}$ and $x_{n}$ in such a way that each ball contains the center of the next one whereas the balls $B\left(x_{i_{k}}, \rho\right)$ are disjoint. This is similar to the classical ball-covering argument, but additional difficulties arise from the necessity to maintain a proper order in the set of balls.

Proof. Let us say that a sequence of indices $\left\{i_{k}\right\}_{k=0}^{l}$ is good if the following conditions are satisfied:

$\left(a^{\prime}\right) 0=i_{0}<i_{1}<\ldots<i_{l}$

$\left(b^{\prime}\right) d\left(x_{i_{k}}, x_{i_{k+1}}\right)<3 \rho$ for all $k=0,1, \ldots, l-1$;

$\left(c^{\prime}\right) d\left(x_{i_{k}}, x_{i_{j}}\right) \geq 2 \rho$ for all distinct $k, j=0,1, \ldots, l$.

Note that a good sequence does not necessarily have $i_{l}=n$ as required in condition $(a)$. We start with a good sequence that consists of a single index $i_{0}=0$, and will successively redefine it to increase at each step the value of $i_{l}$. A terminal good sequence will be used to construct a sequence satisfying $(a),(b),(c)$.

Assuming that $\left\{i_{k}\right\}_{k=0}^{l}$ is a good sequence, define the set of indices

$$
S:=\left\{s: i_{l}<s \leq n \text { and } d\left(x_{s}, x_{i_{k}}\right) \geq 2 \rho \text { for all } k \leq l\right\},
$$

and consider two cases.

Set $S$ is non-empty. In this case we will redefine $\left\{i_{k}\right\}$ to increase $i_{l}$. Let $m$ be the least index in $S$. By (4.29) and the definition of $S, m>i_{l}+1$. Since $m-1$ is not in $S$, we have

$$
d\left(x_{m-1}, x_{i_{k}}\right)<2 \rho \text { for some } k \leq l
$$

(see Figure 2).

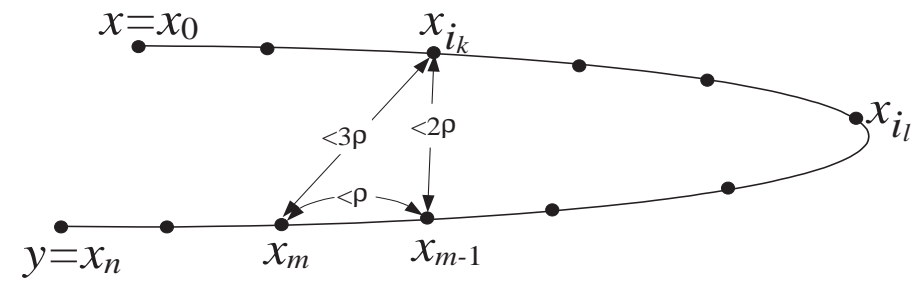

Figure 2.

By (4.30) and $\left(b^{\prime}\right)$ we obtain, for the same $k$ as in (4.30),

$$
d\left(x_{m}, x_{i_{k}}\right) \leq d\left(x_{m}, x_{m-1}\right)+d\left(x_{m-1}, x_{i_{k}}\right)<3 \rho .
$$

Now we modify the sequence $\left\{i_{j}\right\}$ as follows: keep $i_{0}, i_{1}, \ldots, i_{k}$ as before, forget the previously selected indices $i_{k+1}, \ldots, i_{l}$, and set $i_{k+1}:=m$ and $l:=k+1$.

Clearly, the new sequence $\left\{i_{k}\right\}_{k=0}^{l}$ is also good, and the value of $i_{l}$ has increased (although $l$ may have decreased). Therefore, this construction can be repeated only a finite number of times.

Set $S$ is empty. In this case, we will use the existing good sequence to construct a sequence satisfying conditions $(a),(b),(c)$. The set $S$ can be empty for two reasons:

- either $i_{l}=n$, 
- or $i_{l}<n$ and for any index $s$ such that $i_{l}<s \leq n$ there exists $k \leq l$ such that $d\left(x_{s}, x_{i_{k}}\right)<2 \rho$.

In the first case the sequence $\left\{i_{k}\right\}_{k=0}^{l}$ already satisfies $(a),(b),(c)$, and the proof is finished. In the second case, choose the minimal $k \leq l$ such that $d\left(x_{n}, x_{i_{k}}\right)<2 \rho$ (see Figure 3).

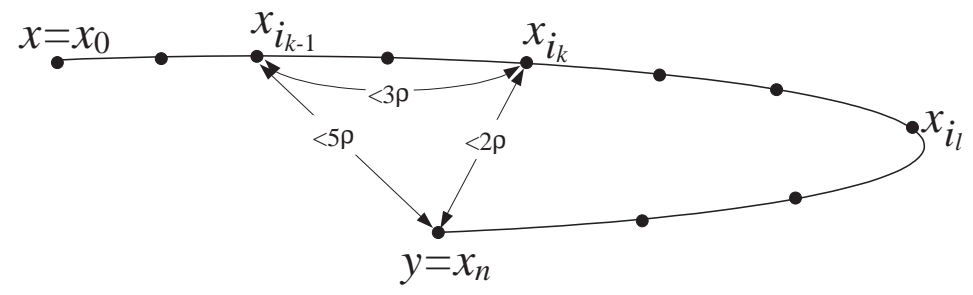

FIGURE 3.

The hypothesis $d\left(x_{n}, x_{0}\right) \geq 2 \rho$ implies $k \geq 1$, and from $\left(b^{\prime}\right)$ we obtain

$$
d\left(x_{n}, x_{i_{k-1}}\right) \leq d\left(x_{n}, x_{i_{k}}\right)+d\left(x_{i_{k}}, x_{i_{k-1}}\right)<5 \rho .
$$

By the minimality of $k$, we also have $d\left(x_{n}, x_{i_{j}}\right) \geq 2 \rho$ for all $j<k$. Hence, we define the final sequence $\left\{i_{j}\right\}$ as follows: keep $i_{0}, i_{1}, \ldots, i_{k-1}$ as before, forget $i_{k}, \ldots, i_{l}$, and set $i_{k}:=n$ and $l:=k$. Then this sequence satisfies $(a),(b),(c)$.

Let $A$ be a subset of $M$ of finite measure; that is, $\mu(A)<\infty$. Then any function $u \in L^{2}(M, \mu)$ is integrable on $A$, and we can set

$$
u_{A}:=\frac{1}{\mu(A)} \int_{A} u d \mu
$$

For any two measurable sets $A, B \subset M$ of finite measure, we have the following identity:

$$
\begin{aligned}
\int_{A} \int_{B}|u(x)-u(y)|^{2} d \mu(x) d \mu(y)= & \mu(A) \int_{B}\left|u-u_{B}\right|^{2} d \mu+\mu(B) \int_{A}\left|u-u_{A}\right|^{2} d \mu \\
& +\mu(A) \mu(B)\left|u_{A}-u_{B}\right|^{2},
\end{aligned}
$$

which is proved by a straightforward computation.

Proof of Theorem 4.8 (ii). The inequality $\beta \leq \alpha+1$ will follow from Corollary 4.3 if we show that for any $\sigma>\frac{\alpha+1}{2}$ the space $W^{\sigma, 2}$ is trivial; that is, $W_{\sigma}(u)<\infty$ implies $u \equiv 0$. To see this, let $x, y$ be two distinct Lebesgue points in $M$. By the chain condition, for any positive integer $n$ there exists a sequence of points $\left\{x_{i}\right\}_{i=0}^{n}$ such that $x_{0}=x, x_{n}=y$, and

$$
d\left(x_{i}, x_{i+1}\right)<C \frac{d(x, y)}{n}:=\rho, \quad \text { for all } 0 \leq i<n .
$$

Assuming that $n$ is large enough so that $d(x, y)>2 \rho$, we obtain by Lemma 4.10 that there exists a subsequence $\left\{x_{i_{k}}\right\}_{k=0}^{l}$ (of course $l \leq n$ ) such that $x_{i_{0}}=x$, $x_{i_{l}}=y$, the balls $\left\{B\left(x_{i_{k}}, \rho\right)\right\}$ are disjoint, and

$$
d\left(x_{i_{k}}, x_{i_{k+1}}\right)<5 \rho,
$$

for all $k=0,1, \ldots, l-1$. 
For simplicity, let $B_{k}:=B\left(x_{i_{k}}, \rho\right), 0 \leq k \leq l-1$. Let $r:=7 \rho<1$. We see that the domain $\left\{(\xi, \eta): \xi \in B_{k}, \eta \in B_{k+1}\right\}$ is contained in the domain $\{(\xi, \eta): \xi \in$ $\left.B_{k}, \eta \in B(\xi, r)\right\}$ for all $0 \leq k \leq l-1$, by (4.32).

By (4.2), (4.31) and (3.2), we have

$$
\begin{aligned}
W_{\sigma}(u) & \geq c r^{-2 \sigma-\alpha} \int_{M} \int_{B(\xi, r)}|u(\xi)-u(\eta)|^{2} d \mu(\eta) d \mu(\xi) \\
& \geq c r^{-2 \sigma-\alpha} \sum_{k=0}^{l-1} \int_{B_{k}} \int_{B_{k+1}}|u(\xi)-u(\eta)|^{2} d \mu(\eta) d \mu(\xi) \\
& \geq c r^{-2 \sigma-\alpha} \rho^{2 \alpha} \sum_{k=0}^{l-1}\left|u_{B_{k}}-u_{B_{k+1}}\right|^{2} \\
& \geq c \rho^{-2 \sigma+\alpha} \frac{1}{l}\left(\sum_{k=0}^{l-1}\left|u_{B_{k}}-u_{B_{k+1}}\right|\right)^{2} \\
& \geq c \rho^{-2 \sigma+\alpha} \frac{1}{n}\left|u_{B_{0}}-u_{B_{l}}\right|^{2} \\
& \geq c \rho^{-2 \sigma+\alpha+1} \frac{\left|u_{B(x, \rho)}-u_{B(y, \rho)}\right|^{2}}{d(x, y)} .
\end{aligned}
$$

Therefore, using $W_{\sigma}(u)<\infty$ and $2 \sigma>\alpha+1$,

$$
\begin{aligned}
|u(x)-u(y)|^{2} & =\lim _{\rho \rightarrow 0}\left|u_{B(x, \rho)}-u_{B(y, \rho)}\right| \\
& \leq C d(x, y) W_{\sigma}(u) \lim _{\rho \rightarrow 0} \rho^{2 \sigma-\alpha-1}=0 .
\end{aligned}
$$

Note that (3.2) implies that $\mu$-almost all points in $M$ are Lebesgue points, and so $u \equiv$ const. Finally, $\mu(M)=\infty$ implies $u \equiv 0$.

4.6. Embedding theorems. In addition to the spaces $L^{p}$ and $W^{\sigma, 2}$ defined above, let us define a Hölder space $C^{\lambda}=C^{\lambda}(M, d, \mu)$ as follows: we say $u \in C^{\lambda}$ if

$$
\|u\|_{C^{\lambda}}:=\|u\|_{\infty}+\underset{\substack{\mu-\operatorname{ess} \sup \\ x, y \in M \\ 0<d(x, y)<1 / 3}}{ } \frac{|u(x)-u(y)|}{d(x, y)^{\lambda}}<\infty .
$$

The restriction $d(x, y)<1 / 3$ here is related to the restriction $r<1$ in the definition (4.1) of the functional $W_{\sigma}(u)$. Note that the $1 / 3$ may be replaced by any other positive constant, giving an equivalent space.

Theorem 4.11. Assume that a metric measure space $(M, d, \mu)$ admits a heat kernel $p_{t}$ satisfying the hypothesis $\left(H_{1}\right)$ (cf. Theorem 4.2). Then the following are true.

(i) If $\alpha>\beta$, then

$$
W^{\beta / 2,2} \hookrightarrow L^{q} \quad \text { for any } 2 \leq q \leq 2^{*}:=\frac{2 \alpha}{\alpha-\beta} .
$$

That is, $u \in W^{\beta / 2,2}$ implies $u \in L^{q}$ and

$$
\|u\|_{q} \leq C\|u\|_{\beta / 2,2} \text {. }
$$

(ii) If $\alpha=\beta$, then

$$
W^{\beta / 2,2} \hookrightarrow L^{q} \quad \text { for any } 2 \leq q<\infty .
$$


(iii) If $\alpha<\beta$, then

$$
W^{\beta / 2,2} \hookrightarrow C^{\lambda} \quad \text { for } \lambda=\frac{\beta-\alpha}{2} .
$$

That is, $u \in W^{\beta / 2,2}$ implies $u \in C^{\lambda}$ and

$$
\|u\|_{C^{\lambda}} \leq C\|u\|_{\beta / 2,2} .
$$

Remark. Observe that the definitions of the function spaces $W^{\beta / 2,2}, L^{q}$, and $C^{\lambda}$ involved in the embedding theorems do not depend on a heat kernel. The existence of a heat kernel is used in the proof in three different ways:

- First, in $(i)$ and $(i i)$ we actually prove the embedding for $\mathcal{D}(\mathcal{E})$ and then use $W^{\beta / 2,2}=\mathcal{D}(\mathcal{E})$, which holds by Theorem 4.2.

- Second, the embedding results for $\mathcal{D}(\mathcal{E})$ in $(i)$ and $(i i)$ explicitly use the estimate

$$
p_{t}(x, y) \leq C t^{-\alpha / \beta} .
$$

- Third, the proof of (iii) uses the estimate (3.2) of the volumes of balls, which holds by Theorem 3.2 . For this part, the hypothesis $\left(H_{1}\right)$ can be replaced by $\left(H_{0}\right)$. Furthermore, $(i i i)$ follows from (3.2) alone, in which case $\beta$ may be any number larger than $\alpha$. With this understanding, the statement of (iii) is not new (see, for example, [6, [15, 21]) and is included for completeness. However, if the hypothesis $\left(H_{1}\right)$ does hold, then it follows from Theorem 4.2 that any function in $\mathcal{D}(\mathcal{E})$ is Hölder continuous.

Remark. If $(M, d)$ satisfies the chain condition (see Definition 3.4), then for any $\lambda>1$ the space $C^{\lambda}$ consists only of constants. On the other hand, by Corollary $4.3 \operatorname{dim} W^{\beta / 2,2}=\infty$, and the embedding (4.35) cannot be true if $\lambda>1$. Hence, by Theorem 4.11( iii) we conclude that $\beta \leq \alpha+2$ (cf. [25]). Of course, by Theorem 4.8 we have the stronger inequality $\beta \leq \alpha+1$ under weaker hypotheses.

Proof. By Theorem 4.2, we have $W^{\beta / 2,2}=\mathcal{D}(\mathcal{E})$ and

$$
\|u\|_{\beta / 2,2} \simeq\|u\|_{2}+\mathcal{E}[u]^{1 / 2}
$$

for any $u \in W^{\beta / 2,2}$. Hence, in parts $(i)$ and (ii), it suffices to prove that

$$
\mathcal{D}(\mathcal{E}) \hookrightarrow L^{q}
$$

(where $2 \leq q \leq 2^{*}$ in case $(i)$ and $2 \leq q<\infty$ in case $(i i)$ ), and

$$
\|u\|_{q} \leq C\left(\|u\|_{2}+\mathcal{E}[u]^{1 / 2}\right)
$$

for any $u \in \mathcal{D}(\mathcal{E})$.

Proof for case (i), $\alpha>\beta$. The upper bound in (1.5) implies that

$$
\mu \text {-ess sup } p_{t, y \in M}(x, y) \leq C t^{-\alpha / \beta} \text {. }
$$

Using the definition (1.3) of the semigroup $T_{t}$, the Cauchy-Schwarz inequality, and the normalization property of the heat kernel, we obtain that for any $u \in L^{2}$, 
$x \in M$, and $t>0$,

$$
\begin{aligned}
\left|T_{t} u(x)\right| & \leq \int_{M} p_{t}(x, y)|u(y)| d \mu(y) \\
& \leq\left\{\int_{M} p_{t}(x, y) u(y)^{2} d \mu(y)\right\}^{1 / 2}\left\{\int_{M} p_{t}(x, y) d \mu(y)\right\}^{1 / 2} \\
& \leq C t^{-\frac{\alpha}{2 \beta}}\|u\|_{2} .
\end{aligned}
$$

In particular, for any $\nu \geq \frac{2 \alpha}{\beta}$, we have

$$
\left\|T_{t} u\right\|_{\infty} \leq C t^{-\frac{\nu}{4}}\|u\|_{2} \text { for all } 0<t<1 .
$$

Hence, the heat semigroup $\left\{T_{t}\right\}$ is $L^{2} \rightarrow L^{\infty}$ ultracontractive for $0<t<1$. Since $\alpha>\beta$, we see that $\nu \geq 2 \alpha / \beta>2$, and so, by [9, Theorem 2.4.2, p.75] (or [7], [28]), $\mathcal{D}(\mathcal{E}) \hookrightarrow L^{\frac{2 \nu}{\nu-2}} ;$ that is, for any $u \in \mathcal{D}(\mathcal{E})$,

$$
\|u\|_{\frac{2 \nu}{\nu-2}}^{2} \leq C \mathcal{E}[u]+C_{0}\|u\|_{2}^{2}
$$

When $\nu$ varies in $\left[\frac{2 \alpha}{\beta},+\infty\right)$, the exponent $q=\frac{2 \nu}{\nu-2}$ varies in $\left(2,2^{*}\right]$. Therefore, (4.42) implies (4.38) for all $2<q \leq 2^{*}$. For the remaining case $q=2$, 4.38 is trivial.

Note that if $\nu=\frac{2 \alpha}{\beta}$, then (4.41) holds for all $t>0$, which implies (4.42) with $C_{0}=0$ (see [9] Corollary 2.4.3]); that is,

$$
\|u\|_{2^{*}}^{2} \leq C \mathcal{E}[u] .
$$

We will need (4.43) in Section 5 .

Proof for case (ii), $\alpha=\beta$. The proof is the same as above, with the following modification. Since (4.41) holds for all $\nu \geq 2 \alpha / \beta=2$, we see that (4.42) holds for all $\nu>2$. Therefore, $q=\frac{2 \nu}{\nu-2}$ takes all the values in $(2,+\infty)$, whence the claim follows.

Proof for case (iii), $\alpha<\beta$. For any $x \in M$ and $r>0$, set

$$
u_{r}(x):=\frac{1}{\mu(B(x, r))} \int_{B(x, r)} u(\xi) d \mu(\xi) .
$$

We claim that for any $u \in W^{\beta / 2,2}$, any $0<r<1 / 3$, and all $x, y \in M$ such that $d(x, y) \leq r$, the following inequality holds:

$$
\left|u_{r}(x)-u_{r}(y)\right| \leq C r^{\lambda} W_{\beta / 2}(u)^{1 / 2} .
$$

Indeed, setting $B_{1}=B(x, r), B_{2}=B(y, r)$, we have

$$
u_{r}(x)=\frac{1}{\mu\left(B_{1}\right)} \int_{B_{1}} u(\xi) d \mu(\xi)=\frac{1}{\mu\left(B_{1}\right) \mu\left(B_{2}\right)} \int_{B_{1}} \int_{B_{2}} u(\xi) d \mu(\eta) d \mu(\xi),
$$

and similarly

$$
u_{r}(y)=\frac{1}{\mu\left(B_{1}\right) \mu\left(B_{2}\right)} \int_{B_{1}} \int_{B_{2}} u(\eta) d \mu(\eta) d \mu(\xi) .
$$


Applying the Cauchy-Schwarz inequality, (3.2) and (4.1), we obtain

$$
\begin{aligned}
\left|u_{r}(x)-u_{r}(y)\right|^{2} & =\left\{\frac{1}{\mu\left(B_{1}\right) \mu\left(B_{2}\right)} \int_{B_{1}} \int_{B_{2}}(u(\xi)-u(\eta)) d \mu(\eta) d \mu(\xi)\right\}^{2} \\
& \leq \frac{1}{\mu\left(B_{1}\right) \mu\left(B_{2}\right)} \int_{B_{1}} \int_{B_{2}}|u(\xi)-u(\eta)|^{2} d \mu(\eta) d \mu(\xi) \\
& \leq C r^{-\alpha} \int_{M}\left[\frac{1}{\mu(B(\xi, 3 r))} \int_{B(\xi, 3 r)}|u(\xi)-u(\eta)|^{2} d \mu(\eta)\right] d \mu(\xi) \\
& \leq C r^{-\alpha+\beta} W_{\beta / 2}(u),
\end{aligned}
$$

thus proving (4.45).

Similarly, one proves that for any $0<r<1 / 3$ and $x \in M$,

$$
\left|u_{2 r}(x)-u_{r}(x)\right| \leq C r^{\lambda} W_{\beta / 2}(u)^{1 / 2} .
$$

Let $x$ be a Lebesgue point of $u$. Setting $r_{k}=2^{-k} r$ for any $k=0,1,2, \ldots$, we obtain from (4.46) that

$$
\begin{aligned}
\left|u(x)-u_{r}(x)\right| & \leq \sum_{k=0}^{\infty}\left|u_{r_{k}}(x)-u_{r_{k+1}}(x)\right| \\
& \leq C\left(\sum_{k=0}^{\infty} r_{k}^{\lambda}\right) W_{\beta / 2}(u)^{1 / 2} \\
& \leq C r^{\lambda} W_{\beta / 2}(u)^{1 / 2} .
\end{aligned}
$$

Applying the Cauchy-Schwarz inequality

$$
\left|u_{r}(x)\right| \leq C r^{-\alpha / 2}\|u\|_{2}
$$

and using (4.47) to some fixed value of $r$, say $r=1 / 4$, we obtain

$$
|u(x)| \leq\left|u(x)-u_{r}(x)\right|+\left|u_{r}(x)\right| \leq C\left(\|u\|_{2}+W_{\beta / 2}(u)^{1 / 2}\right),
$$

whence

$$
\|u\|_{\infty} \leq C\|u\|_{\beta / 2,2} \text {. }
$$

If $y$ is another Lebesgue point of $u$ such that $r:=d(x, y)<1 / 3$, then we obtain from (4.45), (4.47), and a similar inequality for $y$,

$|u(x)-u(y)| \leq\left|u(x)-u_{r}(x)\right|+\left|u_{r}(x)-u_{r}(y)\right|+\left|u_{r}(y)-u(y)\right| \leq C r^{\lambda} W_{\beta / 2}(u)^{1 / 2}$.

Hence,

$$
\frac{|u(x)-u(y)|}{d(x, y)^{\lambda}} \leq C W_{\beta / 2}(u)^{1 / 2},
$$

which together with (4.48) yields (4.36).

Theorem 4.12 (Compact embedding theorem). Let $(M, d, \mu)$ be a metric measure space, and $p_{t}$ be a heat kernel in $M$ satisfying (1.5). Then for any bounded sequence $\left\{u_{k}\right\}$ in $\mathcal{D}(\mathcal{E})$ in the norm (4.7), there exists a subsequence $\left\{u_{k_{i}}\right\}$ that converges to a function $u \in L^{2}(M, \mu)$ in the following sense:

$$
\left\|u_{k_{i}}-u\right\|_{L^{2}(B, \mu)} \rightarrow 0,
$$


for any set $B \subset M$ of finite measure.

Remark. Without any additional assumptions about the functions $\Phi_{1}$ and $\Phi_{2}$, the estimate (1.5) is equivalent to

$$
p_{t}(x, y) \leq C t^{-\alpha / \beta}
$$

and this is exactly what is used in the proof.

Proof. Let $\left\{u_{k}\right\}$ be a bounded sequence in $\mathcal{D}(\mathcal{E})$. Since $\left\{u_{k}\right\}$ is also bounded in $L^{2}$, there exists a subsequence, still denoted by $\left\{u_{k}\right\}$, such that $\left\{u_{k}\right\}$ converges weakly to some function $u \in L^{2}$. Let us show that in fact $\left\{u_{k}\right\}$ converges to $u$ in $L^{2}(B)=L^{2}(B, \mu)$ for any set $B \subset M$ of finite measure.

For any $t>0$, by the triangle inequality we have

$$
\left\|u_{k}-u\right\|_{L^{2}(B)} \leq\left\|u_{k}-T_{t} u_{k}\right\|_{L^{2}(M)}+\left\|T_{t} u_{k}-T_{t} u\right\|_{L^{2}(B)}+\left\|T_{t} u-u\right\|_{L^{2}(M)} .
$$

For any function $v \in L^{2}$ we have

$$
\begin{aligned}
\left\|v-T_{t} v\right\|_{2}^{2} & =\int_{M}\left(\int_{M}(v(x)-v(y)) p_{t}(x, y) d \mu(y)\right)^{2} d \mu(x) \\
& \leq \int_{M}\left\{\int_{M} p_{t}(x, y) d \mu(y) \int_{M}|v(x)-v(y)|^{2} p_{t}(x, y) d \mu(y)\right\} d \mu(x) \\
& =2 t \mathcal{E}_{t}[v] \\
& \leq 2 t \mathcal{E}[v] .
\end{aligned}
$$

Since $\mathcal{E}\left[u_{k}\right]$ is uniformly bounded in $k$ by the hypothesis, we obtain that for all $k$ and $t>0$,

$$
\left\|u_{k}-T_{t} u_{k}\right\|_{2} \leq C \sqrt{t}
$$

Since $\left\{u_{k}\right\}$ converges to $u$ weakly in $L^{2}$ and $p_{t}(x, \cdot) \in L^{2}$, we see that for $\mu$-almost all $x \in M$,

$$
T_{t} u_{k}(x)=\int_{M} p_{t}(x, y) u_{k}(y) d \mu(y) \stackrel{k \rightarrow \infty}{\longrightarrow} \int_{M} p_{t}(x, y) u(y) d \mu(y)=T_{t} u(x) .
$$

Also, by (4.40) we have

$$
\left\|T_{t} u_{k}\right\|_{\infty} \leq C t^{-\frac{\alpha}{2 \beta}}\left\|u_{k}\right\|_{2}
$$

so that the sequence $\left\{T_{t} u_{k}\right\}$ is uniformly bounded in $k$ for any $t>0$. Since $\left\{T_{t} u_{k}\right\}$ converges to $T_{t} u$ almost everywhere, the dominated convergence theorem yields

$$
T_{t} u_{k} \stackrel{k \rightarrow \infty}{\longrightarrow} T_{t} u \quad \text { in } \quad L^{2}(B),
$$

because $\mu(B)<\infty$. Hence, we obtain from (4.49), (4.50), and 4.51) that for any $t>0$,

$$
\limsup _{k \rightarrow \infty}\left\|u_{k}-u\right\|_{L^{2}(B)} \leq C \sqrt{t}+\left\|T_{t} u-u\right\|_{L^{2}(M)} .
$$

Since $T_{t} u \rightarrow u$ in $L^{2}(M)$ as $t \rightarrow 0$, we finish the proof by letting $t \rightarrow 0$.

Corollary 4.13. Let $(M, d, \mu)$ be a metric measure space, and let $p_{t}$ be a heat kernel in $M$ satisfying (1.5) with $\Phi_{1}(1)>0$. Then for any bounded sequence $\left\{u_{k}\right\}$ in $\mathcal{D}(\mathcal{E})$ in the norm (4.7), there exists a subsequence $\left\{u_{k_{i}}\right\}$ that converges to a function $u \in L^{2}(M, \mu)$ almost everywhere. 
Proof. By the first part of the proof of Theorem [3.2, the hypothesis $\Phi_{1}(1)>0$ implies finiteness of the measure of any ball. Fix a point $x \in M$ and consider the sequence of balls $B_{N}=B(x, N)$, where $N=1,2, \ldots$ By Theorem 4.12 we can assume that the sequence $\left\{u_{k}\right\}$ converges to $u \in L^{2}(M)$ in the norm of $L^{2}\left(B_{N}\right)$ for any $N$. Therefore, there exists a subsequence that converges almost everywhere in $B_{1}$. From this sequence, let us select a subsequence that converges to $u$ almost everywhere in $B_{2}$, and so on. Using the diagonal principle, we obtain a subsequence that converges to $u$ almost everywhere in $M$.

\section{Semilinear elliptic equations}

As above, let $(M, d, \mu)$ be a metric measure space that possesses a heat kernel satisfying (1.5). In this section we show the existence of generalized solutions of the equation

$$
-\mathcal{L} u+f(x, u)=g(x),
$$

where $\mathcal{L}$ is the Laplace operator in $M$ defined by (4.3) or (4.8). More precisely, we say that $u \in \mathcal{D}(\mathcal{E})$ is a generalized solution of (5.1) if the following identity holds:

$$
\mathcal{E}(u, v)+\int_{M} f(x, u(x)) v(x) d \mu(x)-\int_{M} g(x) v(x) d \mu(x)=0,
$$

for any test function $v$ from a certain class to be defined below.

Fix a couple $p, q \in(1, \infty)$, set

$$
E^{p, q}:=\mathcal{D}(\mathcal{E}) \cap L^{p} \cap L^{q},
$$

and define the norm in $E^{p, q}$ by

$$
\|u\|:=\|u\|_{p}+\|u\|_{q}+\mathcal{E}[u]^{1 / 2} .
$$

Clearly $E^{p, q}$ is a Banach space, and its dual is

$$
\left(E^{p, q}\right)^{*}=E^{p^{\prime}, q^{\prime}}
$$

where $p^{\prime}$ and $q^{\prime}$ are the Hölder conjugates to $p$ and $q$, respectively.

We assume throughout this section that

$$
g \in L^{p^{\prime}}
$$

and

$$
|f(x, u)| \leq C|u|^{q-1}+f_{0}(x), \quad \text { for all } x \in M \text { and } u \in \mathbb{R},
$$

where $f_{0}$ is a non-negative function in $L^{q^{\prime}}$.

Let us show that all the terms in (5.2) make sense if $u \in \mathcal{D}(\mathcal{E}) \cap L^{q}$ and $v \in E^{p, q}$. Indeed, $\mathcal{E}(u, v)$ is defined as $u, v \in \mathcal{D}(\mathcal{E})$, and the other two terms are finite by the Hölder inequality:

$$
\left|\int_{M} g v d \mu\right| \leq\|g\|_{p^{\prime}}\|v\|_{p}<\infty
$$

and

$$
\left|\int_{M} f(\cdot, u) v d \mu\right| \leq\|f(\cdot, u)\|_{q^{\prime}}\|v\|_{q} \leq\left(C\|u\|_{q}^{q / q^{\prime}}+\left\|f_{0}\right\|_{q^{\prime}}\right)\|v\|_{q}<\infty .
$$

Now we can give a precise definition of a generalized solution of (5.1).

Definition 5.1. Assuming that $f$ and $g$ satisfy (5.3) and (5.4), we say that $u \in E^{p, q}$ is a generalized solution of (5.1) if the identity (5.2) holds for all $v \in E^{p, q}$. 
Let $E$ be a Banach space and $I: E \rightarrow \mathbb{R}$ be a functional on $E$. Recall that $I$ is Fréchet differentiable at $u \in E$ if there exists an element in the dual space $E^{*}$ of $E$, denoted by $I^{\prime}(u)$, such that for all $v \in E$,

$$
I(u+t v)=I(u)+t I^{\prime}(u) v+o(t) \quad \text { as } t \rightarrow 0 .
$$

The functional $I^{\prime}(u)$ is called the Fréchet derivative of $I$ at point $u$. We say that $I$ is continuously Fréchet differentiable if $I$ is Fréchet differentiable at any $u \in E$, and the mapping $u \mapsto I^{\prime}(u)$ is a continuous mapping from $E$ to $E^{*}$ (see, for example, [19], [24]). Finally, we say that $u$ is a critical point of $I$ if $I^{\prime}(u)=0$.

We will show that a generalized solution of (5.1) may be obtained as a critical point of a functional $I(u)$, where $I(u)$ is defined by

$$
I(u):=\frac{1}{2} \mathcal{E}[u]+\int_{M} F(x, u(x)) d \mu(x)-\int_{M} g(x) u(x) d \mu(x),
$$

and

$$
F(x, u):=\int_{0}^{u} f(x, s) d s .
$$

Let us show that the functional $I$ defined by (5.7) is continuously Fréchet differentiable for suitable $f$ and $g$.

Proposition 5.2. Assume that $f(x, u)$ is continuous in $u \in \mathbb{R}$ for all $x \in M$ and satisfies (5.4), and $g$ satisfies (5.3). Then I defined as in (5.7) is continuously Fréchet differentiable in $E^{p, q}$. Moreover, we have

$$
I^{\prime}(u) v=\mathcal{E}(u, v)+\int_{M} f(x, u(x)) v(x) d \mu(x)-\int_{M} g(x) v(x) d \mu(x),
$$

for all $u, v \in E^{p, q}$.

Thus, $u$ is a generalized solution of (5.1) if and only if $u$ is a critical point of $I$.

Proof. The proof follows the same line as in [19]. For completeness, we sketch the proof. It is easy to see directly from the definition that the functional

$$
I_{0}(u)=\frac{1}{2} \mathcal{E}[u]-\int_{M} g(x) u(x) d \mu(x)
$$

is continuously Fréchet differentiable at any point $u \in E^{p, q}$, and

$$
I_{0}^{\prime}(u) v=\mathcal{E}(u, v)-\int_{M} g(x) v(x) d \mu(x) .
$$

Let us show that the remaining part of $I$, namely the functional

$$
J(u):=\int_{M} F(x, u(x)) d \mu(x),
$$

is also continuously Fréchet differentiable, and

$$
J^{\prime}(u) v=\int_{M} f(x, u(x)) v(x) d \mu(x) .
$$

Indeed, taking (5.10) as the definition of $J^{\prime}$, we have, for all $u, v \in E^{p, q}$ and $-1<$ $t<1$,

$$
\begin{aligned}
J(u+t v)-J(u)-t J^{\prime}(u) v & =\int_{M}\left[\int_{u}^{u+t v} f(x, s) d s-t f(x, u(x)) v(x)\right] d \mu(x) \\
& =t \int_{M}(f(x, u+\theta v)-f(x, u)) v(x) d \mu(x)
\end{aligned}
$$


where

$$
\theta=\theta(x, t) \in[0, t] \subset(-1,1) .
$$

By (5.6) we have $f(\cdot, u) v \in L^{1}$. By (5.4) and (5.6) we obtain, similarly,

$$
|f(\cdot, u+\theta v)| \leq C|u+\theta v|^{q-1}+f_{0} \leq C(|u|+|v|)^{q-1}+f_{0} \in L^{1} .
$$

Since $f(x, u+\theta v) \rightarrow f(x, u)$ as $t \rightarrow 0$, we conclude by the dominated convergence theorem that

$$
J(u+t v)-J(u)-t J^{\prime}(u) v=o(t) \quad \text { as } t \rightarrow 0,
$$

proving that $J$ is Fréchet differentiable.

It remains to show that $J^{\prime}(u)$ is continuous. For any $u_{1}, u_{2}, v \in E^{p, q}$, we have

$$
\begin{aligned}
\left|J^{\prime}\left(u_{1}\right) v-J^{\prime}\left(u_{2}\right) v\right| & =\left|\int_{M}\left(f\left(x, u_{1}(x)\right)-f\left(x, u_{2}(x)\right)\right) v(x) d \mu(x)\right| \\
& \leq\left\|f\left(\cdot, u_{1}\right)-f\left(\cdot, u_{2}\right)\right\|_{q^{\prime}}\|v\|_{q} \\
& \leq\left\|f\left(\cdot, u_{1}\right)-f\left(\cdot, u_{2}\right)\right\|_{q^{\prime}}\|v\|_{E^{p, q}},
\end{aligned}
$$

whence

$$
\left\|J^{\prime}\left(u_{1}\right)-J^{\prime}\left(u_{2}\right)\right\|_{\left(E^{p, q}\right)^{*}} \leq\left\|f\left(\cdot, u_{1}\right)-f\left(\cdot, u_{2}\right)\right\|_{q^{\prime}} .
$$

Note that the Nemytsky operator $\mathcal{F} u:=f(x, u(x))$ is continuous from $L^{q}$ to $L^{q^{\prime}}$, provided that $f$ satisfies (5.4) (see [27, Theorem 19.1]; for a bounded domain, see [1, Theorem 2.2]). Indeed, if $\mathcal{F} u$ is not continuous, then there exists a sequence $\left\{u_{k}\right\}$ such that $\left\|u_{k}-u\right\|_{q} \rightarrow 0$ but

$$
\left\|\mathcal{F} u_{k}-\mathcal{F} u\right\|_{q^{\prime}} \geq \varepsilon
$$

for all $k$ and some $\varepsilon>0$. Since $\left\{u_{k}\right\}$ converges to $u$ in $L^{q}$, there is a subsequence $\left\{u_{k_{j}}\right\}$ of $\left\{u_{k}\right\}$ such that $\left\{u_{k_{j}}\right\}$ converges to $u$ almost everywhere in $M$. Fix some $R>0$ and set $B_{R}=B\left(x_{0}, R\right)$ for a fixed point $x_{0} \in M$. By (5.4) and the dominated convergence theorem, we have

$$
\begin{aligned}
& \lim _{j \rightarrow \infty}\left\|\mathcal{F} u_{k_{j}}-\mathcal{F} u\right\|_{q^{\prime}}^{q^{\prime}} \\
& \quad=\lim _{j \rightarrow \infty}\left\{\int_{B_{R}}\left|f\left(\cdot, u_{k_{j}}\right)-f(\cdot, u)\right|^{q^{\prime}} d \mu+\int_{M \backslash B_{R}}\left|f\left(\cdot, u_{k_{j}}\right)-f(\cdot, u)\right|^{q^{\prime}} d \mu\right\} \\
& \quad \leq C \lim _{j \rightarrow \infty} \int_{M \backslash B_{R}}\left(\left|u_{k_{j}}\right|^{q}+|u|^{q}+\left|f_{0}\right|^{q^{\prime}}\right) d \mu \\
& \quad=C \lim _{j \rightarrow \infty} \int_{M \backslash B_{R}}\left(\left|u_{k_{j}}-u+u\right|^{q}+|u|^{q}+\left|f_{0}\right|^{q^{\prime}}\right) d \mu \\
& \quad \leq C \int_{M \backslash B_{R}}\left(|u|^{q}+\left|f_{0}\right|^{q^{\prime}}\right) d \mu .
\end{aligned}
$$

Choosing $R$ large enough, we can make the right-hand side arbitrarily small, which contradicts (5.11). Hence, $\mathcal{F} u$ is continuous from $L^{q}$ to $L^{q^{\prime}}$. Therefore, $J^{\prime}(u)$ is continuous.

By Proposition 5.2, in order to prove the existence of a generalized solution of (5.1), it is enough to show that the functional $I$ defined by (5.7) has a critical point in $E^{p, q}$; this in turn will follow if $I$ has a minimum point in $E^{p, q}$. The following 
statement provides the conditions that ensure that a functional on a Banach space has a minimum point.

Proposition 5.3 ([19, Theorem 2.5, p.14]). Let I be a real-valued functional in a reflexive Banach space $E$ satisfying the following conditions:

(i) I is bounded below; that is,

$$
\inf _{u \in E} I(u)>-\infty .
$$

(ii) $I$ is coercive; that is, for any real a there exists $b$ such that $I(u) \leq a$ implies $\|u\| \leq b$.

(iii) Any sequence $\left\{u_{k}\right\}$ that converges to $u$ weakly in $E$ has a subsequence $\left\{u_{k_{i}}\right\}$ such that

$$
\liminf _{i \rightarrow \infty} I\left(u_{k_{i}}\right) \geq I(u) .
$$

Then I has a minimum point in $E$.

Now we prove the main result of this section.

Theorem 5.4. Assume that a metric measure space $(M, d, \mu)$ admits a heat kernel $p_{t}$ satisfying the hypothesis $\left(H_{1}\right)$ (cf. Theorems 4.2, 4.11), and assume that $\alpha>\beta$. Fix two real numbers $p, q$ such that

$$
q \geq p \geq 2^{*}:=\frac{2 \alpha}{\alpha-\beta} .
$$

Let $g \in L^{p^{\prime}}$, and let $f(x, u)$ be a measurable function on $M \times \mathbb{R}$ that is continuous in $u$ for any $x \in M$ and satisfies (5.4). Moreover, assume that, for all $x \in M$ and $u \in \mathbb{R}$,

$$
F(x, u):=\int_{0}^{u} f(x, s) d s \geq c|u|^{q}+F_{0}(x),
$$

where $F_{0} \in L^{1}$. Then (5.1) has a generalized solution $u \in E^{p, q}$.

Remark. Here is an example of a function $f$ that satisfies all the hypotheses of Theorem 5.4:

$$
f(x, u)=|u|^{q-2} u \text {. }
$$

Hence, the equation

$$
-\mathcal{L} u+|u|^{q-2} u=g
$$

has a generalized solution $u \in E^{p, q}$ for any $g \in L^{p^{\prime}}$, provided $p$ and $q$ satisfy (15.12). We will see below that this solution is unique.

Proof. It suffices to show that the functional $I$ defined by (5.7) satisfies conditions (i) - (iii) of Proposition 5.3 .

Condition (i): $I$ is bounded below. By (5.12) there exists $\theta \in[0,1]$ such that

$$
\frac{1}{p}=\frac{\theta}{2^{*}}+\frac{1-\theta}{q},
$$

whence, for any $u \in E^{p, q}$,

$$
\|u\|_{p} \leq\|u\|_{2^{*}}^{\theta}\|u\|_{q}^{1-\theta}
$$

by the Hölder inequality. By (4.43), we see that

$$
\|u\|_{2^{*}} \leq C \mathcal{E}[u]^{1 / 2},
$$


which implies

$$
\begin{aligned}
\|u\|_{p} & \leq C \mathcal{E}[u]^{\theta / 2}\|u\|_{q}^{1-\theta} \\
& \leq C\left(\|u\|_{q}+\mathcal{E}[u]^{1 / 2}\right) .
\end{aligned}
$$

From (5.7), (5.13), (5.5) and (5.15), we obtain that, for any $u \in E^{p, q}$,

$$
\begin{aligned}
I(u) & \geq \frac{1}{2} \mathcal{E}[u]+\left(c\|u\|_{q}^{q}-\left\|F_{0}\right\|_{1}\right)-\|g\|_{p^{\prime}}\|u\|_{p} \\
& \geq \frac{1}{2} \mathcal{E}[u]+c\|u\|_{q}^{q}-\left\|F_{0}\right\|_{1}-C\|g\|_{p^{\prime}}\left(\|u\|_{q}+\mathcal{E}[u]^{1 / 2}\right) \\
& \geq\left[\frac{1}{2} \mathcal{E}[u]-C \mathcal{E}[u]^{1 / 2}\right]+\left[c\|u\|_{q}^{q}-C\|u\|_{q}\right]-C \\
& \geq\left[\frac{1}{2} s^{2}-C s\right]+\left[c t^{q}-C t\right]-C,
\end{aligned}
$$

where $s:=\mathcal{E}(u, u)^{1 / 2}$ and $t:=\|u\|_{q}$. Since $q>1$, the value of each square bracket is bounded below, whence we conclude that $I$ is bounded below.

Condition (ii): $I$ is coercive. If $I(u) \leq a$ for some $a$, then by (5.16),

$$
a \geq\left[\frac{1}{2} s^{2}-C s\right]+\left[c t^{q}-C t\right]-C,
$$

which implies that $s$ and $t$ must be bounded. Together with (5.15), this implies that $u$ is bounded in $E^{p, q}$.

Condition (iii). Let a sequence $\left\{u_{k}\right\}$ converge to $u$ weakly in $E^{p, q}$. Since $g \in$ $L^{p^{\prime}} \subset\left(E^{p, q}\right)^{*}$, we have

$$
\lim _{k \rightarrow \infty} \int_{M} g u_{k} d \mu=\int_{M} g u d \mu .
$$

Since $u \in \mathcal{D}(\mathcal{E}) \subset\left(E^{p, q}\right)^{*}$, we have

$$
\lim _{k \rightarrow \infty} \mathcal{E}\left(u_{k}, u\right)=\mathcal{E}[u] .
$$

Applying the inequality

$$
\mathcal{E}\left[u_{k}\right] \geq 2 \mathcal{E}\left(u_{k}, u\right)-\mathcal{E}[u],
$$

we obtain

$$
\liminf _{k \rightarrow \infty} \mathcal{E}\left[u_{k}\right] \geq \mathcal{E}[u] .
$$

We are left to verify that there exists a subsequence $\left\{u_{k_{i}}\right\}$ such that

$$
\liminf _{i \rightarrow \infty} \int_{M} F\left(\cdot, u_{k_{i}}\right) d \mu \geq \int_{M} F(\cdot, u) d \mu .
$$

The sequence $\left\{u_{k}\right\}$ is bounded in $\mathcal{D}(\mathcal{E})$. Therefore, by Corollary 4.13 there exists a subsequence $\left\{u_{k_{i}}\right\}$ that converges to $u$ almost everywhere in $M$. Therefore, we have also $F\left(\cdot, u_{k_{i}}\right) \rightarrow F(\cdot, u)$ almost everywhere in $M$. By (5.13), we have $F\left(\cdot, u_{k_{i}}\right) \geq F_{0} \in L^{1}$, and (5.19) follows by Fatou's lemma. Combining (5.17)-(5.19), we complete the proof.

Finally, we complement Theorem 5.4 by a uniqueness result. 
Proposition 5.5. Let the function $f(x, u)$ be strictly monotone increasing in $u$ for every $x \in M$. Then the equation (5.1) has at most one generalized solution $u \in E^{p, q}$.

In particular, the equation (5.14) has exactly one generalized solution in $E^{p, q}$.

Proof. Let $u_{1}$ and $u_{2}$ be two generalized solutions of (5.1). Then for any $v \in E^{p, q}$ we have from (5.2),

$$
\mathcal{E}\left(u_{1}-u_{2}, v\right)+\int_{M}\left(f\left(\cdot, u_{1}\right)-f\left(\cdot, u_{2}\right)\right) v d \mu=0 .
$$

Substituting $v=u_{1}-u_{2}$, we obtain

$$
\mathcal{E}\left[u_{1}-u_{2}\right]+\int\left(f\left(\cdot, u_{1}\right)-f\left(\cdot, u_{2}\right)\right)\left(u_{1}-u_{2}\right) d \mu=0 .
$$

By the monotonicity of $f(x, u)$ in $u$, both terms here are non-negative, and so each of them must vanish. In particular, we obtain

$$
\left(f\left(\cdot, u_{1}\right)-f\left(\cdot, u_{2}\right)\right)\left(u_{1}-u_{2}\right)=0
$$

almost everywhere, which by the strict monotonicity of $f$ implies $u_{1}=u_{2}$.

\section{REFERENCES}

1. A. Ambrosetti and G. Prodi, A primer of nonlinear analysis, Cambridge University Press, 1993. MR 94f:58016

2. N. Aronszajn and K. T. Smith, Theory of Bessel potentials. part 1, Ann. Inst. Fourier, Grenoble 11 (1961), 385-475. MR 26:1485

3. M. T. Barlow, Diffusions on fractals, Lectures on Probability Theory and Statistics, vol. 1690, Lecture Notes Math., Springer-Verlag, 1998, pp. 1-121. MR 2000a:60148

4. W Which values of the volume growth and escape time exponents are possible for graphs?, preprint (2001)

5. M. T. Barlow and R. F. Bass, Brownian motion and harmonic analysis on Sierpiński carpets, Canadian J. Math. 51 (1999), 673-744. MR 2000i:60083

6. M. Biroli and U. Mosco, Sobolev and isoperimetric inequalities for Dirichlet forms on homogeneous spaces, Rend. Mat. Acc. Lincei VI (1) (1995), 37-44. MR 96i:46034a

7. E. A. Carlen, S. Kusuoka, and D. W. Stroock, Upper bounds for symmetric Markov transition functions, Ann. Inst. Henri Poincaré-Probab. Statist. 23 (1987), 245-287. MR 88i:35066

8. E. B. Davies, One-parameter semigroups, Academic Press, 1980. MR 82i:47060

9. _ Heat kernels and spectral theory, Cambridge University Press, 1990. MR 92a:35035

10. K. J. Falconer, Fractal geometry-mathematical foundation and applications, John Wiley, 1992. MR 92j:28008

11. P. J. Fitzsimmons, B. M. Hambly, and T. Kumagai, Transition density estimates for Brownian motion on affine nested fractals, Commun. Math. Phys. 165 (1994), 595-620. MR 95j:60122

12. M. Fukushima, Y. Oshima Y., and M. Takeda, Dirichlet forms and symmetric Markov processes, Studies in Mathematics, 19, De Gruyter, 1994. MR 96f:60126

13. A. Grigor'yan, Estimates of heat kernels on Riemannian manifolds, London Math. Soc. Lecture Note Ser., vol. 273, 1999, pp. 140-225. MR 2001b:58040

14. A. Grigor'yan and A. Telcs, Sub-Gaussian estimates of heat kernels on infinite graphs, Duke Math. J. 109 (2001), 451-510.

15. P. Hajłasz and P. Koskela, Sobolev met Poincaré, Mem. Amer. Math. Soc. 145 (2000), no. 688. MR 2000j: 46063

16. E. Hille and R. S. Phillips, Functional analysis and semi-groups, Amer. Math. Soc., 1957. MR 19:664d

17. J. Hu and K. S. Lau, Riesz potentials and Laplacians on fractals, preprint (2001).

18. A. Jonsson, Brownian motion on fractals and function spaces, Math. Zeit. 222 (1996), 495504. MR 97e:60137 
19. I. Kuzin and S. Pohozaev, Entire solutions of semilinear elliptic equations, Birkhäuser, 1997. MR 99d:35050

20. P. Li and S. T. Yau, On the parabolic kernel of the Schrödinger operator, Acta Math. 156 (1986), 153-201. MR 87f:58156

21. R. A. Macías and C. Segovia, Lipschitz functions on spaces of homogeneous type, Adv. Math. 33 (1979), 257-270. MR 81c:32017a

22. K. Pietruska-Pałuba, Some function spaces related to the Brownian motion on simple nested fractals, Stochastics and Stochastics Reports 67 (1999), 267-285. MR 2000h:60078

23. - On function spaces related to fractional diffusions on d-sets, Stochastics and Stochastics Reports 70 (2000), 153-164. MR 2001i:60123

24. P. R. Rabinowitz, Minimax methods in critical point theory with applications to differential equations, Expository Lectures from CBMS Regional Conference held at University of Miami, Amer. Math. Soc., Providence, RI, 1986. MR 87j:58024

25. A. Stós, Symmetric $\alpha$-stable processes on d-sets, Bull. Pol. Acad. Sci. Math. 48 (2000), 237245. MR 2002f:60152

26. A. Telcs, Random walks on graphs, electric networks and fractals, J. Probability Theory and Related Fields 82 (1989), 435-449. MR 90h:60065

27. M. M. Vainberg, Variational methods for the study of nonlinear operators, San Francisco: Holden-Day, Inc., 1964. MR 31:638

28. N. Th. Varopoulos, Hardy-Littlewood theory for semigroups, J. Funct. Anal. 63 (1985), 240260. MR 87a:31011

29. K. Yosida, Functional analysis, Springer-Verlag, 1980. MR 82i:46002

Department of Mathematics, Imperial College, London, SW7 2BZ, United Kingdom and The Institute of Mathematical Sciences, The Chinese University of Hong Kong, Shatin, N.T., Hong Kong

E-mail address: a.grigoryan@ic.ac.uk

Department of Mathematical Sciences, Tsinghua University, Beijing 100084 China and Department of Mathematics, The Chinese University of Hong Kong, Shatin, N.T., HONG KONG

E-mail address: jxhu@math.tsinghua.edu.cn

Department of Mathematics, The Chinese University of Hong Kong, Shatin, N.T., HONG KONG

E-mail address: kslau@math.cuhk.edu.hk 